\begin{tabular}{|c|c|c|c|c|c|}
\hline MUNIBE Antropologia-Arkeologia & $n^{0} 71$ & $5-21$ & DONOSTIA & 2020 & ISSN 1132-2217 • eISSN 2172-4555 \\
\hline
\end{tabular}

\title{
Tafonomía del registro fósil de macromamíferos del Pleistoceno Superior en el nivel 4 de la Cueva de Prado Vargas (Cornejo, Burgos, España)*
}

\section{Taphonomy of the fossil record of macromammals of the Upper Pleistocene at level 4 of the Cueva de Prado Vargas (Cornejo, Burgos, Spain)*}

PALABRAS CLAVES: Paleolítico medio, Zooarqueología, Tafonomía.

GAKO-HITZAK: Erdi Paleolitoa, Zooarkeologia, Tafonomia.

KEY WORDS: Middle Palaeolithic, Zooarchaeology, Faunal Taphonomy.

Héctor DE LA FUENTE JUEZ(a), Sandra GARCíA DOMÍNGUEZ ${ }^{(b)}$ Marta NAVAZO RUIZ(c), Ruth BLASCO LÓPEZ(d)

\section{RESUMEN}

Las técnicas de subsistencia en grupos humanos del pasado se investigan en profundidad a través del estudio de las acumulaciones fósiles, de su formación, origen y composición. Los conjuntos faunísticos localizados en los yacimientos arqueológicos pueden reflejar el tipo de estrategias desarrolladas por los homínidos a través de disciplinas como la Zooarqueología y la Tafonomía. El presente trabajo analiza el conjunto faunístico del Nivel 4 de la Cueva de Prado Vargas (Cornejo, Burgos, España) con una antigüedad de 46.400 años BP (MIS 3). Este yacimiento se ha interpretado como lugar de ocupación recurrente donde procesaron los recursos cárnicos los grupos neandertales que habitan la zona sur de la Cordillera Cantábrica, quienes intercalan su presencia con otros predadores que aprovechan su ausencia en la cavidad para dejar su huella en el registro.

\section{LABURPENA}

Metaketa fosilen, eraketaren, jatorriaren eta osaeraren azterketa eginda modu sakonean ikertzen dira iraganeko giza taldeen iraupen-teknikak. Aztarnategi arkeologikoetan topatutako multzo faunistikoek hominidoek garatutako estrategia mota isla dezakete hainbat diziplina bidez: zooarkeologiaren eta tafonomiaren bidez, esate baterako. Lan honek Prado Vargaseko (Cornejo, Burgos, Espainia) kobazuloko 4. mailako multzo faunistikoa aztertzen du. Aipatutako aztarnategiak 46.400 BP urteko antzinatasuna (MIS 3) du. Aztarnategi horrek okupazio errepikakorra izan zuela uste da. Bertan prozesatu zituzten haragizko baliabideak Kantauriar mendikatearen hegoaldean bizi ziren talde Neanderthalek, baina haien presentzia beste harrapakari batzuenarekin ere tartekatu zen. Izan ere, barrunbe hartan ez zeuden unea aprobetxatu zuten erregistroan haien aztarna uzteko.

\section{ABSTRACT}

As predators, humans used to transport part of their prey to safe places like caves. Not only these accumulations, but also their formation, origin and composition constitute a fundamental basis to explore the behaviour of the human groups and their interaction with environment. Zooarchaeology and Taphonomy are complementary disciplines that allow us to approach these objectives. In this study, the faunistic assemblage of level 4 of Prado Vargas cave (Cornejo, Burgos, España) is analysed. This site is localised between the Cantabrian cornice and the north Meseta with a dating of 46.000 years BP. This work focuses on the livelihood strategies and Neanderthal behaviour, especially the way in which their prey was exploited. The analysis of the existing marks on the bones tells us about access to external tissues (meat, tendons or skin), processing and consumption, as well as bone for other non-nutritive purposes. They also allow us to explore and the way of access to animals, allowing us to distinguish the ways of life groups of prehistoric hunters-gatherers. Furthermore, we focus on differentiating the agent responsible for the fracture, as well as the state of the bone at the time that this occurred studying the patterns of fracturing and the marks associated with the percussion, as well as different tafonomic processes. We have collected a series of data that are offered in this work. The level 4 of Prado Vargas has been interpreted as a place of recurring habitat for Neanderthals, who intersperse their presence with other predators who take advantage of their absence in the cavity to leave their mark on the record.

\footnotetext{
* Esta publicación se enmarca en el proyecto financiado por la Junta de Castilla y León desde la Consejería de Cultura y Turismo, Dirección General de Patrimonio Cultural, "Intervención arqueológica en la Cueva de Prado Vargas, en Cornejo (Merindad de Sotoscueva, Burgos)" (C02W06).

(a) Universidad de Burgos, Facultad de Humanidades y Comunicación. Dpt. De Historia, Geografía y Comunicación. Área de prehistoria. Calle Paseo de los Comendadores S/N. 09001 Burgos. Correo: hector13scott@gmail.com https://orcid.org/0000-0002-8882-5132.

(b) Universidad de Burgos, Facultad de Humanidades y Comunicación. Dpt. De Historia, Geografía y Comunicación. Área de prehistoria. Calle Paseo de los Comendadores S/N. 09001 Burgos. Correo: sgd0026@gmail.com.

(c) Universidad de Burgos, Facultad de Humanidades y Comunicación. Dpt. De Historia, Geografía y Comunicación. Área de prehistoria. Calle Paseo de los Comendadores S/N. 09001 Burgos. Centro Nacional de Investigación en Evolución Humana (CENIEH). Paseo Sierra de Atapuerca, 3, Burgos. Correo: mnavazo@ubu.es https://orcid.org/0000-0002-3602-2741.

(d) Centro Nacional de Investigación en Evolución Humana (CENIEH). Paseo Sierra de Atapuerca, 3, Burgos. Correo: ruth.blasco@cenieh.es https://orcid.org/0000-0001-9804-739X.
} 


\section{INTRODUCCIÓN}

Las evidencias antropogénicas en los conjuntos faunísticos nos permiten explorar el grado de interacción entre los grupos humanos y la fauna del entorno, incluyendo las relaciones con otros predadores, como los carnívoros, siendo las modificaciones de ambos agentes biológicos la base para la interpretación de muchos yacimientos paleolíticos (e.g. Binford, 1981, 1988; Blumenschine, 1995; Blumenschine et al., 2007; Bunn y Kroll, 1986, 1988; Egeland et al., 2004; Marean y Kim, 1998; Selvaggio, 1994, 1998). Las estrategias de subsistencia de las sociedades pretéritas han suscitado grandes debates en la comunidad científica, especialmente el método de obtención de los recursos faunísticos (e.g. Álvarez et al., 1992; Binford, 1981, 1989; Brain, 1981; Chase, 1986, 1989; Castaños, 2005; Costamagno et al., 2006; Díez et al., 2008; Eixea et al., 2012; Gabucio et al., 2014, 2017; Gaudzinski y Roebroeks, 2000; Klein y Cruz-Uribe, 1984; Luret et al., 2020; Rendu et al; 2012; Romandini et al., 2014; Rosell et al., 2012; Sánchez-Hernández et al., 2014; Stiner, 1994; Villaverde et al., 2017; Yravedra, 2006a; Yravedra Sainz De Los Terreros y Cobo, 2015). Existe un gran abanico de posibilidades entre la caza más activa y el carroñeo más pasivo, aunque la tendencia actual es usar términos en función del tipo (primario o secundario) y modo de acceso (activo o pasivo) y el tiempo de intervención (inmediato o tardío), existiendo distintos tipos de captación según las oportunidades que se sostengan (Bunn y Ezzo, 1993). O'Connell et al. (1988) recoge que lejos de tabús de comportamiento, los homínidos de todos los periodos no han desechado la posibilidad de aprovechar un cadáver fresco si la ocasión surge, no indicando este carroñero unas menores aptitudes cinegéticas. La importancia de la ubicación territorial, la estacionalidad, el tipo de presa y su procesado, la abundancia de restos y las modificaciones tafonómicas ayudan a interpretar el comportamiento de los homínidos, permitiendo superar el viejo debate entre cazadores-carroñeros (e.g. Conard y Prindville, 2000).

Uno de los puntos esenciales para conocer la historia de un yacimiento es analizar los procesos de rotura del conjunto óseo (Johnson, 1985). Ésta puede deberse a diferentes agentes y procesos que se combinan a lo largo de la formación del conjunto. Varios estudios han demostrado que las alteraciones óseas pueden ser diferentes en función del estado del hueso en el momento de su fracturación (e.g. Cáceres et al., 2002; Johnson, 1985; Karr, 2015; Karr y Outram, 2012; Morla, 1984; Outram, 2002; Pickering y Egeland, 2006; Tersigni, 2007; Villa y Mahieu, 1991). Las diferencias estriban principalmente en la pérdida de hidratación, la cual afecta en gran medida a la capacidad del hueso para absorber y soportar estrés, alterando su microestructura (Johnson, 1985; Tersigni, 2007). La rotura ósea se debe a diferentes agentes y procesos que se combinan a lo largo de la historia de un conjunto. Discernir el tipo de fractura que generan los agentes biológicos y distinguirla de aquella provocada por procesos fosildiagenéticos ha sido el foco de atención de numerosos investigadores (e.g. Bartram y Marean, 1999; Binford, 1981; Brain, 1981; Bunn, 1983, 1989; Klein y Cruz-Uribe, 1984; Johnson, 1985; Outram, 2001; Villa y Mahieu, 1991). De forma general, la fracturación en fresco posee unas características morfológicas determinadas (Johnson, 1985; Morlan, 1980; Villa y Mahieu, 1991) que, junto a elementos diagnósticos de intencionalidad en la acción, indican acceso a la médula (e.g. Cáceres, 2002; Díez et al., 1999; Fernández-Jalvo, 1999). Sin embargo, este objetivo es perseguido tanto por homínidos como por carnívoros, quienes pueden llegar a realizar morfologías muy similares. Para asignar la fracturación a uno de ellos y secuenciar la acción de ambos agentes es necesario tener en cuenta otros rasgos diagnósticos, como las alteraciones que produce la fracturación antrópica (e.g. Blumenschine y Selvaggio, 1988; Blumenschine, 1995; Domínguez-Rodrigo y Barba, 2006; Pickering y Egeland, 2006; Karr y Outram, 2012; Merritt y Davis, 2017; Moclan y Domínguez-Rodrigo, 2018), otras alteraciones generadas tras la carnicería, como las marcas de corte (e.g. Binford, 1981; Blumenschine y Selvaggio, 1988; Bromage y Boyde, 1984; Capaldo y Blumenschine, 1994; Domínguez-Rodrigo et al., 2009, 2017; Fisher, 1995; Potts y Shipman, 1981; Shipman y Rose, 1983) u otros daños provocados por carnívoros, como las mordeduras, entre otras alteraciones (e.g. Blumenchine, 1991; Blumenschine y Selvaggio, 1988; Bunn, 1981; Capaldo, 1995; Maguire et al., 1980; Selvaggio, 1994; Stiner, 1994; Rosell y Blasco, 2009). La exposición a los agentes atmosféricos trae consigo la incidencia de los rayos UV y cambios de humedad y temperatura que afectan también a la estructura del hueso provocando la ruptura de las fibras de colágeno, produciéndose astillamientos, agrietaciones y exfoliaciones en la superficie de los restos (Behrensemeyer, 1978; Bromage, 1985; Fernández Jalvo, 1992). El aumento de la fragilidad permite que los huesos se rompan con facilidad. El pisoteo puede producir cierto grado de fragmentación y pequeñas marcas en los bordes de fractura (Agenbroad, 1989; Blasco et al., 2008; Gifford-González, 1989; Irving et al., 1989). La fragmentación por pisoteo se produce con mayor facilidad en restos afectados por la meteorización, que les hace más propensos a fragmentarse en las áreas de debilidad en respuesta a la presión ejercida; por el contrario, en los huesos en estado fresco solo mostrarán estrías como efecto del pisoteo (Fiorillo, 1989; Haynes, 1991; Olsen y Shipman, 1988). Se debe tener en cuenta también, que la fragmentación por caída de bloques si puede ocasionar puntos de impacto, pero no patrones repetitivos (Cáceres, 2002).

Dentro del marco tan amplio que ofrece la tafonomía aplicada a la zooarqueología se encuadra este estudio, mediante el cual se pretende abordar la problemática que afecta al conjunto faunístico del nivel 4 de la cueva de Prado Vargas, arrojando luz sobre cuestiones 
referentes a la obtención y el procesado de los recursos cárnicos o la interacción de los homínidos, tanto con el medio en el que se desarrollan sus actividades, como con el resto de depredadores con los que entran en competencia directa por los recursos animales.

\section{EL SITIO DE PRADO VARGAS}

La Cueva de Prado Vargas se localiza en el norte de la Península Ibérica, en la localidad de Cornejo (Burgos) formando parte del complejo kárstico de Ojo Guareña. La cavidad se sitúa a 20 m sobre la margen derecha del río Trema y cuenta unos 120 m de desarroIlo plano y lineal, con una altura constante inferior a dos metros. El nivel intervenido hasta el momento, durante las campañas de 1986, 2006, 2016 y 2017, es el Nivel 4, que ha sido datado por racemización de aminoácidos en un premolar de équido dando como resultado 46,4 ka (Navazo et al., 2005; Navazo y Díez, 2008).

Se ha recuperado durante las campañas 1986, 2016 y 2017 un total de 820 piezas. La materia prima predominante es el sílex cretácico de origen local que supone más del 90\% del conjunto, seguido muy de lejos por cuarcita y lutita cuyo origen todavía no hemos encontrado. Se encuentran representadas todas las categorías de la cadena operativa, percutores (4\%); núcleos (8\%); lascas (72\%), lascas retocadas (12\%), y fragmentos indeterminables (4\%). La explotación es predominantemente bifacial discoide y está encaminada a la obtención de soportes de pequeño y mediano tamaño., que se funcionalizan para el aprovechamiento cárnico, trabajo de piel y en algún caso para trabajo vegetal (Santamaría et al., 2016). Llama la atención el grado de aprovechamiento de los núcleos, que se reciclan en ocasiones a útiles una vez que están agotados. Dentro del conjunto de lascas retocadas, destaca el retoque simple, siendo los tipos más representados raederas y denticulados

El conjunto faunístico localizado en el Nivel 4 de la Cueva de Prado Vargas se caracteriza principalmente por la presencia de ungulados, siendo el más abundante el Cervus elaphus, también se localizan Equus ferus, Bos/Bison, Capra pyrenaica, Rupicapra pyrenaica, Sus scrofa, Oryctolagus sp. y tres especies de carnívoros, Ursus spelaeus, Canis lupus y Meles meles, cuya presencia es esporádica.

\section{METODOLOGÍA}

Los restos faunísticos del Nivel 4 de la Cueva de Prado Vargas se han clasificado a nivel taxonómico y anatómico. Los restos que no han podido ser identificados a nivel de especie o familia, se han atribuido a categorías por tallas de peso basadas en estudios africanos (e.g. Bunn, 1986; Bunn et al., 1988) y adaptados en trabajos posteriores para yacimientos europeos (Cáceres, 2002): talla muy grande (> 1000kg), talla grande (300-1000kg), talla media (100-300kg), talla pequeña (20-100kg) y talla muy pequeña $(<20 \mathrm{~kg})$. Para determinar la edad de muerte, se han empleado principalmente el reemplazo y desgaste dentario (e.g. Azorit et al., 2002). Para la cuantificación del registro se ha tenido en cuenta el NR (Número de Restos), NISP (Número de Especímenes Identificados), NME (Número Mínimo de Elementos) y NMI (Número Mínimo de Individuos) a partir de la repetición de partes anatómicas teniendo en cuenta el lado que ocupa el hueso en el esqueleto y la edad de los animales (Lyman, 1994; Schmid, 1972). También se ha calculado el Índice de Supervivencia anatómica (ISu): \% supervivencia = MNE x 100/ número de elementos en el esqueleto del animal $\times$ MNI, que permite valorar la integridad de la muestra de los elementos recuperados. Asimismo, se ha empleado a partir de un coeficiente de correlación $r$ de Pearson la posibilidad de una destrucción diferencial de los restos óseos según su densidad (Lam et al., 1999; Lyman, 1985). Por último, se ha utilizado el Índice Modificado de Utilidad General (MGUI) (Binford, 1978) y el Índice de Utilidad (UI) (Emerson, 1993).

Las alteraciones óseas superficiales se han analizado a nivel macro y microscópico. Para el análisis microscópico hemos empleado una lupa binocular modelo MOTIC SMZ-140 Series. Las marcas de corte se han examinado teniendo en cuenta el tipo (incisión, aserrado y tajo), la disposición y zona del hueso donde se localiza la marca, su orientación, delineación y medidas (Fernández-Jalvo y Andrews, 2016; Lyman, 2008; Shipman y Rose, 1983). Las modificaciones producidas por carnívoros se han clasificado como: depresiones, surcos, improntas, vaciados de las epífisis, bordes crenulados, furrowing, digestión y pitting (Binford, 1981). Se han analizado según la región anatómica y tejido óseo (esponjoso o cortical) donde se localizan, tomando datos sobre su orientación y medidas en el caso de las mordeduras (Blumenschine, 1995; Fernández-Jalvo y Andrews, 2016; Haynes, 1980, 1983) y se han tenido en cuenta la representación anatómica, taxonómica y edad de muerte de los animales representados en el registro. Las medidas de estas marcas se han comparado posteriormente con los estudios realizados por varios autores (Andrews y Jalvo, 1997; Arilla et al., 2014; Delaney-Rivera et al., 2009; Domínguez-Rodrigo y Piqueras, 2003; Selvaggio y Wilder, 2001; Yravedra, 2004).

Para conocer el estado de los huesos en el momento de su rotura, se han analizado los planos de fractura según los criterios establecidos por Villa y Mahieu (1991). Teniendo en cuenta para su análisis la delineación (transversal, longitudinal o curvada), el ángulo (oblicuo, recto o mixto) y la superficie de fractura (suave o irregular). Además, se han analizado la sección diafisaria y se han tomado medidas de la longitud. En total se establecieron 5 porciones numéricas: 1) Epífisis proximal 2) Metáfisis proximal 3) Diáfisis 4) Metáfisis distal 5) Epífisis distal. Así mismo se ha registrado a la cara del hueso a la que se corresponden. 
El proceso de fracturación antrópica produce una serie de características identificables, como pueden ser estigmas de percusión, muescas de percusión, conos (incluyendo lascas medulares y corticales), esquirlas parásitas, contragolpes (Blasco et al., 2013; Blumenschine y Selvaggio, 1988; Capaldo y Blumenschine, 1994; Pickering y Egeland, 2006) y peeling (White, 1992). Respecto a las muescas de percusión, se han analizado teniendo en cuenta su situación con respecto al fragmento óseo, documentando la cara y la porción donde se localizan.

También se han identificado las diferentes coloraciones sobre los restos óseos alterados mediante acción térmica, respondiendo a una serie de variables donde influyen principalmente la temperatura adquirida durante el proceso y el tiempo de exposición al mismo (e.g. Cáceres, 2002; Gifford-Gonzalez, 1989; Shipman et al., 1984). Para este estudio se han seguido los patrones macroscópicos de Stiner et al. (1995), estableciendo seis grados de cremación, incluyendo el grado 0 como ausencia de alteración. Así mismo se han analizado otros procesos postdeposicionales como el weathering (Behrensmeyer, 1978), el trampling (Blasco et al., 2008), la abrasión (Beherensmeyer, 1990; Brett, 1990 y Lyman 1994; Cáceres, 1995), la presión sedimentaria (Shipman, 1981), la humedad (Coard, 1999; Courty et al., 1989; Fernández-Jalvo y Andrews, 2000) y las acción de las raíces (Beherensmeyer, 1978; Cáceres, 2002; Fernández-Jalvo, 1992).

\section{RESULTADOS}

El Nivel 4 de Prado Vargas ha proporcionado hasta la fecha un total de 1566 elementos. Se han clasificado 190 (12,1\%) elementos a nivel taxonómico, mientras que el resto se ha clasificado por categorías de tamaño debido a la alta fragmentación de los restos. Los fósiles identificados más abundantes pertenecen a Cervus elaphus (113), seguido de Capra pyrenaica (19) y Equus ferus (14). Por otra parte, se han documentado un mínimo de 22 individuos en el conjunto, pertenecientes a varias edades predominando fundamentalmente la edad adulta (Tabla 1). Según la estimación de la edad en los restos dentales, Arceredillo (2015) propuso una ocupación acontecida durante el invierno y la primavera, época en las que especies rupícolas como rebecos y cabras, descienden de las montañas para refugiarse en zonas forestales.

Con el cálculo del ISu, se observa una representación esquelética sesgada en todas las tallas del conjunto analizado (Tabla 2). La muestra se caracteriza principalmente por la presencia del esqueleto apendicular (estilopodios, zeugopodios y metapodios). Según los índices de utilidad general, se aprecia cómo en todas las tallas el volumen medular es el valor más elevado debido a la presencia principal de estilopodios y zeugopodios en el conjunto. El valor de la grasa es bajo en todas las tallas, especialmente en las tallas grandes y

\begin{tabular}{|c|c|c|c|c|c|c|c|}
\hline & & & & & \multirow{2}{*}{$\begin{array}{l}\text { NMI Edad } \\
\text { Inmaduro }\end{array}$} & \multirow[b]{2}{*}{ Adulto } & \multirow[b]{2}{*}{ Seni } \\
\hline & NR & NISP & NME & NMI & & & \\
\hline Bos/Bison & 6 & 6 & 5 & 1 & & 1 & \\
\hline Equus ferus & 14 & 14 & 13 & 3 & 1 & 2 & \\
\hline Cervus elaphus & 113 & 113 & 36 & 3 & 1 & 2 & \\
\hline Caprinae & 7 & 7 & 2 & & & & \\
\hline R. pyrenaica & 11 & 11 & 9 & 3 & 1 & 2 & \\
\hline C. pyrenaica & 19 & 19 & 16 & 3 & & 3 & \\
\hline Sus scrofa & 1 & 1 & 1 & 1 & 1 & & \\
\hline Oryctolagus sp. & 2 & 2 & 2 & 2 & 1 & 1 & \\
\hline Ursus spelaeus & 7 & 7 & 7 & 2 & 1 & & 1 \\
\hline Canis lupus & 7 & 7 & 7 & 3 & 1 & 2 & \\
\hline Meles meles & 3 & 3 & 3 & 1 & & 1 & \\
\hline Talla muy grande & 1 & & 1 & & & & \\
\hline Talla grande & 99 & & 22 & & & & \\
\hline Talla media & 346 & & 26 & & & & \\
\hline Talla pequeña & 838 & & 46 & & & & \\
\hline Talla muy pequeña & 11 & & 11 & & & & \\
\hline No identificado & 81 & & & & & & \\
\hline TOTAL & 1566 & 190 & 207 & 22 & 7 & 14 & 1 \\
\hline
\end{tabular}

Tabla 1: NR, NISP, MNE y MNI del conjunto óseo del Nivel 4 de la cueva de Prado Vargas. / NR, NISP, MNE and MNI from Level 4 faunal assemblage of Prado Vargas Cave.

medias, por lo que la mayor parte de elementos consumidos no estarían única y exclusivamente relacionados con la selección de grasa. El esqueleto axial apenas tiene representación, siendo más abundante en las tallas pequeñas (20-100 kg). El esqueleto craneal, representado especialmente por mandíbulas se documenta en todas las categorías de peso, especialmente entre las tallas medias. En los huesos largos, la diáfisis es la parte más abundante $(81,9 \%)$, seguido de las metáfisis (14,7\%) y finalmente, las epífisis (3,2\%). Los resultados según el índice de correlación entre las densidades óseas e ISu, muestran que el Nivel 4 apenas presenta conservación diferencial en función de la densidad ósea; sólo es posible apreciar una débil correlación para los animales de talla media. Aun así, estos valores no son suficientes para argumentar la ausencia de ciertos elementos esqueléticos en el depósito.

Se han analizado un total de 5187 paños de fractura, siendo los ángulos oblicuos (80,72\%), las delineaciones curvadas $(44,57 \%)$ y las superficies suaves (96,68\%) las características predominantes (Fig.1). Estos porcentajes son similares en todas las tallas estudiadas. En cuanto a la longitud, el 96,48\% muestran menos de dos porciones, y respecto a la sección diafisaria representada, el 76,02\% contiene menos de 2 caras. Se han identificado 136 muescas (8,68\%) (Fig. 2), 35 estigmas de percusión (2,23\%), 75 conos de percusión $(4,79 \%)$ y dos casos de peeling, además en 6 casos se han localizado muescas junto a estigmas. Merece la pena destacar que el $8,62 \%$ de las muescas se 


\begin{tabular}{|c|c|c|c|c|c|c|}
\hline & Densidad caballo & \%lsu Talla grande & Densidad ciervo & \%lsu talla media & Densidad oveja & \%lsu talla pequeña \\
\hline Mandíbula & 60,00 & 25,00 & 57,00 & 66,67 & 55,00 & 42,86 \\
\hline Vertebras & 54,00 & 0,96 & 30,00 & 1,28 & 26,00 & 1,10 \\
\hline Costillas & 55,00 & 0,00 & 40,00 & 1,28 & 37,00 & 2,20 \\
\hline Pelvis & 65,00 & 12,50 & 49,00 & 16,67 & 49,00 & 14,29 \\
\hline Escapula & 67,00 & 12,50 & 49,00 & 33,33 & 33,00 & 14,29 \\
\hline Húmero & 64,00 & 62,50 & 63,00 & 83,33 & 42,00 & 42,86 \\
\hline Radio & 84,00 & 50,00 & 68,00 & 83,33 & 52,00 & 50,00 \\
\hline Ulna & 65,00 & 25,00 & 45,00 & 50,00 & 26,00 & 14,29 \\
\hline Fémur & 59,00 & 62,50 & 57,00 & 66,67 & 36,00 & 57,14 \\
\hline Tibia & 82,00 & 50,00 & 74,00 & 83,33 & 59,00 & 28,57 \\
\hline Metacarpo & 84,00 & 25,00 & 72,00 & 66,67 & 67,00 & 7,14 \\
\hline Metatarso & 91,00 & 0,00 & 74,00 & 83,33 & 68,00 & 0,00 \\
\hline Falanges & 67,00 & 0,00 & 57,00 & 6,94 & 55,00 & 1,79 \\
\hline rde Pearson & & 0,121700694 & & 0,822475574 & & $-0,039386975$ \\
\hline
\end{tabular}

Tabla 2: Índice de correlación en densidades máximas de elementos anatómicos y la tasa de supervivencia esquelética según las tallas. Talla grande (Lam et al., 1999), Talla media (Lyman, 1985) y Talla pequeña (Lyman, 1985). / Correlation index in maximum densities of anatomical elements and weight sizes from skeletal survival rate. Large size (Lam et al., 1999), Medium sized (Lyman, 1985) and Small size (Lyman, 1985).

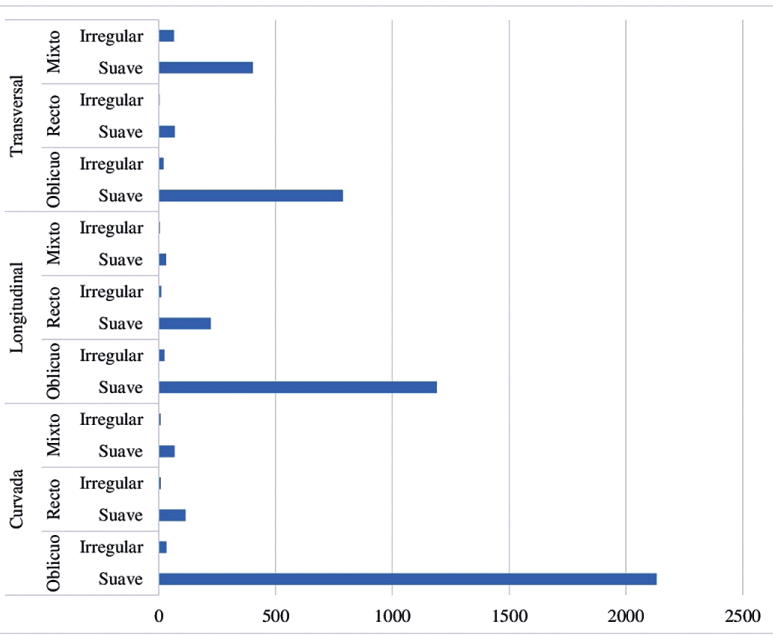

Fig.1. Clasificación de los paños de fractura según los criterios establecidos por Villa y Mahieu (1991). / Classification of fracture shapes on bone following Villa y Mahieu (1991).

localizan opuestas y el $72,99 \%$ de ellas se encuentran aisladas. En cuanto a su disposición en la zona del hueso, el 95,80\% se hallan en la diáfisis, con preferencias en la cara anterior y posterior (Tabla 3).

Por lo que respecta a las marcas de corte, en el Nivel 4 hemos localizado un total de 294 (18,7\%) huesos con esta modificación, concentrándose principalmente en huesos largos de talla pequeña (Tabla A.1.). Las marcas de corte más comunes en el conjunto estudiado son las incisiones (89\%) con orientaciones tanto oblicuas como transversales al eje del hueso. La situación de las marcas de corte en elementos esqueléticos muestra la secuencia de consumo animal, apreciando marcas de descarnación de grandes masas musculares en forma de largas incisiones $(N R=288)$ y raspado

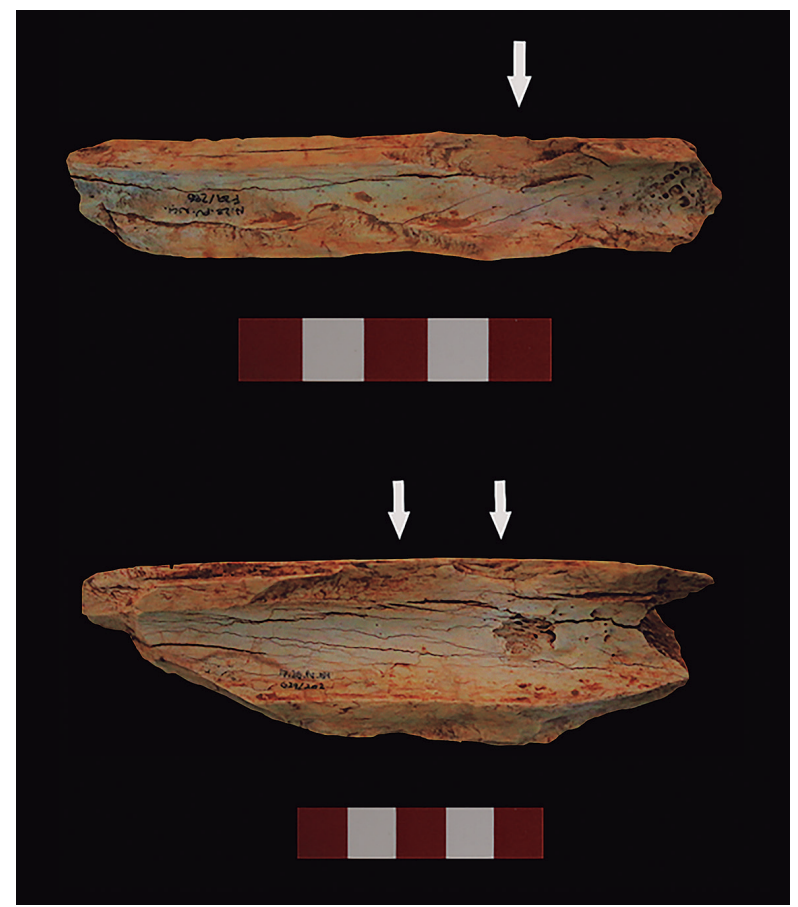

Fig.2. Elementos diagnósticos de fractura intencional. Podemos observar en el fragmento superior una muesca aislada, mientras que en el fragmento interior observamos dos muescas consecutivas. / Diagnostic elements of intentional fracture. We could see in the top of the image an isolated percussion notch, while at the bottom we could see two consecutive percussion notches.

de periostio en forma de raspados $(N R=5)$. La mayor parte de marcas de corte se localizan en las diáfisis de los huesos largos (95,9\%). La termoalteración también está presente en el Nivel 4 de Prado Vargas en el 5\% de los restos ( $N R=79)$, siendo el estadio 4 el más abundante (coloraciones grisáceas). Los restos calcinados 


\begin{tabular}{|l|c|c|c|c|c|c|c|c|c|}
\hline & Ant & Post & Int & Ext & Lat & Ant (Lat) & Ant (Ext) & Post (Int) & Post (ext) \\
\hline Tallas & & & & & & & & & \\
\hline Talla Pequeña & 5 & 3 & 1 & 0 & 2 & 0 & 0 & 0 & 0 \\
\hline Talla Media & 6 & 7 & 5 & 4 & 4 & 1 & 1 & 1 & 1 \\
\hline Talla Grande & 3 & 2 & 1 & 2 & 1 & 0 & 0 & 0 & 0 \\
\hline Elemento anatómico & & & & & & & & & \\
\hline Huesos largos & 1 & 0 & 0 & 0 & 0 & 0 & 0 & 0 & 0 \\
\hline Metacarpo & 3 & 0 & 0 & 0 & 0 & 0 & 0 & 0 & 0 \\
\hline Radio & 1 & 1 & 0 & 0 & 1 & 0 & 0 & 0 & 0 \\
\hline Húmero & 2 & 5 & 1 & 4 & 1 & 1 & 0 & 1 & 0 \\
\hline Metatarso & 2 & 1 & 1 & 0 & 1 & 0 & 0 & 0 & 0 \\
\hline Tibia & 2 & 3 & 4 & 2 & 0 & 0 & 0 & 0 & 1 \\
\hline Fémur & 3 & 2 & 1 & 2 & 2 & 1 & 0 & 0 & 0 \\
\hline
\end{tabular}

Tabla 3: Impactos encontrados en la diferentes tallas y elementos anatómicos. Se emplearon las siguientes abreviaturas: Ant (Anterior), Post (Posterior), Int (Interior), Ext (Exterior) y Lat (Lateral). Cuando encontramos una fuera y otra entre paréntesis, quiere decir que pertenece a la primera pero que tiene tendencia hacia la segunda. / Impacts found in the different weigh sixes and anatomical elements. We use the next abbreviations: Ant (Anterior). Post (Posterior), Int (Interior), Ext (Exterior) y Lat (Lateral). When we found one of them out and other in brackets, we mean that the face in brackets tend to other which is out of brackets.

de este nivel se caracterizan por tener dimensiones inferiores a $50 \mathrm{~mm}$ de longitud que solo son superados en dos de los casos.

Los carnívoros son el otro principal agente biológico que interviene en el conjunto, generando mordeduras y fracturas, principalmente sobre el esqueleto apendicular. Se han localizado un total de 133 restos óseos $(8,4 \%)$ con modificaciones realizadas por carnívoros, entre los que se distinguen depresiones y surcos $(N R=111)$, bordes crenulados $(N R=7)$, cilindros diafisarios $(N R=2)$, digestión $(N R=6)$ y pitting $(N R=7)$. Las dimensiones de las mordeduras sobre cortical dura varían entre 7,4 y 0,2 mm de longitud y 3,8 y 0,1 $\mathrm{mm}$ de anchura. Las medidas tomadas sobre restos óseos de cortical blanda se sitúan entre 5,8 y 0,4 mm de longitud y 2,6 y 0,5 mm de anchura. Utilizando las medidas proporcionadas por varios autores (Andrews y Jalvo, 1997; Arilla et al., 2014; Delaney-Rivera et al., 2009; Domínguez-Rodrigo y Piqueras, 2003; Selvaggio y Wilder, 2001; Yravedra, 2004), nuestros datos parecen coincidir con un gran abanico de depredadores. No obstante, el rango de dimensiones de mordeduras encaja con los carnívoros que están registrados en la cueva (Ursus spelaeus, Canis lupus y Meles meles), aunque no se puede descartar la actuación de otros predadores. Sin embargo, cabe reseñar que la morfología y las dimensiones de las mordeduras tienen limitaciones, siendo resolutivos solamente para determinar el tipo y el tamaño del depredador (Domínguez-Rodrigo y Piqueras, 2003; Yravedra et al., 2017a), aunque en los últimos años esta problemática se ha ido superando a partir de micro-fotogrametría, morfometría geométrica y el uso de machine learning algorithms, permitiendo diferenciar agentes específicos (e.g. Arriaza et al., 2017, 2019; Domínguez-Rodrigo y Baquedano, 2018; Yravedra et al., 2017a, 2017b).
El conjunto de alteraciones fosildiagenéticas, su recuento, su disposición, tipología y región del hueso a la que pertenecen, se han recogido en la Tabla A.2. Entre ellas destacan el trampling $(77,45 \%)$, las fisuras $(73,17 \%)$, el pulido $(80,17 \%)$ y el manganeso $(89,03 \%)$.

\section{DISCUSIÓN}

En el Nivel 4 se documenta una selección sistemática de partes anatómicas con alto valor nutricional y medular, marcas asociadas a descarnación de grandes paquetes musculares y un predominio de individuos adultos. Todos estos elementos sugieren que el acceso antrópico a las presas pudo ser mayoritariamente primario, pudiendo desarrollar técnicas cinegéticas reiteradas (e.g. Enloe, 1991; Conard y Prindiville, 2000; Grayson y Delpech, 2003; Gaudzinski, 2006; Gaudzinski y Niven, 2009; Rendu 2010; Daujeard y Moncel, 2010; Niven at al., 2012; Rendu et al., 2012; Kindler at al., 2014).

A partir de los taxones recuperados se estima que la dieta cárnica de los grupos neandertales que habitaba la cavidad era amplia, comprendiendo varios tipos de presas de distintas tallas de peso. La especialización en un tipo de animal concreto no es una característica definitoria en el nivel estudiado, a pesar de existir predilección por los ungulados de talla pequeña y media. El mayor porcentaje de restos se localiza en las tallas pequeñas sin identificar, suponiendo un 53,5\% del total del conjunto estudiado. Estos datos indican que animales del tamaño de Capra pyrenaica o Rupicapra pyrenaica, pudieron ser los más consumidos por estos grupos humanos. En un marco geográfico relativamente próximo y con cronologías similares observamos unas estrategias de caza basadas en ungulados entre los que destacan los caprinos en Venta Laperra (Ca- 
rranza, Vizcaya), el nivel C de Axlor o en el nivel Smk-I de Arrillor (Zigoitia, Álava) (Baldeón, 1999; Castaños, 2005; González et al. 2005). Algo similar observamos en contextos más lejanos como Cova Negra y Cova Beneito, dónde los neandertales del MIS 3 realizaron una caza no especializada basada en algunos caprinos, además de ciertos ungulados (Salazar-García et al., 2015). Sin embargo, no debemos perder de vista la importancia del Cervus elaphus en el nivel estudiado, dado que los elevados porcentajes de restos de ciervo en yacimientos como Covalejos (Piélagos, Cantabria), El Castillo (Puente Viesgo, Cantabria), los niveles Musterienses XI-XII y XVLa de El Pendo o el nivel 15 de Cueva Morin explicarían la importancia de este taxón en su dieta (Altuna, 1971, 1973; Castaños, 2005; Fuentes Vidarte, 1980; Luret et al., 2020; Pike-Tay et al., 1999). Los restos identificados como Oryctolagus sp. y otros elementos clasificados como "talla muy pequeña", no presentan evidencias carniceras y se recuperaron completos. Esto contrasta con lo encontrado en la Cova del Bolomor, donde los lagomorfos parecen ser consumidos habitualmente por los homínidos, dado que identifican la intervención antropogénica del procesamiento a partir de marcas de corte, rotura intencional, marcas dentales humanas y patrones de quemado (Blasco y Fernández-Peris, 2012; Blasco et al., 2013; Sanchís Serra y Fernández-Peris, 2008). La identificación de explotación de este tipo de pequeñas presas (e.g., lagomorfos, aves, recursos acuáticos, etc.) resulta compleja debido a las pocas marcas que genera su manipulación. Sin embargo, en los niveles Solutrense (III) y Musteriense (IV) de Gorhams Cave se tomó como agente acumulador principal el antrópico por la ausencia de trazas significativas de predación no humana, además de por la elevada fragmentación del conjunto (Brown et al., 2011). Los carnívoros también podrían haber introducido restos de lagomorfos al interior de la cavidad, aunque por lo general, los carnívoros de tallas medias y grandes que consumen presas de talla muy pequeña fracturan de forma acusada los huesos e incluso los ingieren casi completamente (e.g. Horwitz 1990; Pickering, 2001; Tappen y Wrangham, 2000). Si bien es cierto que en el yacimiento de Riparo Mochi (Italia), encontramos tooth marks de pequeños carnívoros en huesos de lagomorfos que evidencian su acceso (Stiner, 2002). También otra opción podría ser la intrusión natural de los propios animales en la cueva debido a sus conductas fosadoras, sin embargo, no se han localizado conexiones anatómicas o un elevado porcentaje de individuos inmaduros que nos permitan apoyar esta hipótesis.

\subsection{El transporte de las canales}

El transporte diferencial identificado comprende prácticamente todas las tallas de peso, así pues, los primeros pasos de la secuencia de procesamiento se realizan en el lugar de obtención de la presa, donde procesan el cadáver para trasladar a la cavidad las partes más atrayentes para estos grupos humanos. Asimismo, también se han documentado restos craneales en el Nivel 4, sugiriendo también el aprovechamiento de otras vísceras. En estos casos, se podría formular que no toda la evisceración se produce tras el abatimiento de la presa, sino que pudo realizarse posteriormente en el lugar de ocupación, especialmente en el caso de las tallas pequeñas. En casos puntuales, también se registran costillas o vértebras al interior de la cavidad, pero apenas cuentan con evidencias de procesamiento antrópico. Se debe tener en cuenta que las marcas sobre estos elementos, especialmente en la cara interna de las costillas, se relacionan con la evisceración. El hecho de no localizar marcas sobre esta parte del hueso no significa que no se produjera esta actividad, ya que la frecuente utilización de las manos provocaría su infrarrepresentación.

\subsection{El aprovechamiento de los recursos animales}

En el área de ocupación, los grupos humanos procesan las presas obtenidas realizando actividades como la descarnación o la fracturación de huesos para acceder a la médula. Generalmente, la descarnación y la desarticulación son actividades que se realizan de forma simultánea en la cadena operativa faunística (Binford, 1978, 1981, 1984; Potts y Shipman, 1981; Shipman y Rose, 1983). La extracción de grandes masas musculares genera una serie de daños característicos en forma de incisiones longitudinales y oblicuas en las diáfisis de huesos largos. Tras la extracción de las grandes masas cárnicas, se tiende a recuperar la carne adherida al hueso, produciendo aserrados transversales y oblicuos generalmente localizados en las metáfisis de huesos largos. Tras la descarnación, se produce el raspado de periostio, cuyo objetivo es facilitar la fracturación de los huesos (Binford, 1981, 1984; Potts y Shipman, 1981; Shipman y Rose, 1983, 1984). Sin embargo, otros autores no ligan estas marcas a la extracción del periostio, sino al aprovechamiento de restos de carne adheridos al hueso (Cáceres, 2002) o a cortes con el filo oblicuo, si la anchura es más reducida y aparecen incisiones asociadas (Delpech y Villa, 1992).

Los datos obtenidos tras el análisis del conjunto revelan una desproporción entre la representación de epífisis y diáfisis de huesos largos. Esta disposición no parece estar relacionada con la conservación del esqueleto en función de la densidad mineral ósea, de modo que la ausencia de epífisis en el conjunto atiende a otra causa. Esta infrarrepresentación podría estar relacionada con factores biológicos, como los grupos humanos o los carnívoros que acceden a la cavidad. Algunos autores sugieren que la ausencia de epífisis se produce por una separación sistemática de estas porciones para aprovechar la grasa que contienen los tejidos esponjosos. Este fenómeno ha sido investigado tanto en yacimientos de la Prehistoria reciente, como en grupos de cazadores-recolectores actuales (e.g. 
Binford, 1978). El debate sobre la extracción de grasa de la materia esponjosa del hueso a través del hervido por neandertales se lleva produciendo desde hace unos años sin alcanzar consenso (Costamagno, 2013; Speth, 2012). Otra de las posibilidades que se maneja en la literatura, es la desaparición de los fragmentos esponjosos como consecuencia de su uso como combustible o como una forma de eliminación de residuos (Perlès y Masset, 1977; Théry-Parisot y Meignen, 1998; Théry-Parisot, 2002). El empleo de restos óseos como combustible es documentado en varios periodos como reflejo de las elevadas proporciones de grasa que contienen los tejidos esponjosos (Théry-Parisot y Meignen, 1998, Théry- Parisot, 2002). Cáceres (2002) demuestra que el empleo conjunto de hueso fresco, especialmente epífisis, y madera como combustible es más efectivo que el uso exclusivo de madera. Esta autora destaca además que "los huesos por sí solos no son buenos combustibles y, por tanto, necesitan un mínimo de combustible leñoso para quemar". Cabe destacar que el hueso puede ser arrojado al fuego no solo cómo combustible sino como práctica higiénica (Costamagno et al. 2005; Morin 2010; Yravedra y Uzquiano, 2013). Por otro lado, los carnívoros a menudo visitan las cavidades en épocas de ausencia humana como consecuencia de sus propias características etológicas y/o para aprovechar los restos abandonados por los grupos humanos, por lo tanto, otra de las hipótesis podría estar también relacionada con la acción de estos predadores en su intento de aprovechar los nutrientes de los restos. Varios estudios sobre las características etológicas de los carnívoros (Haynes, 1980; 1983; Blumenschine, 1988; Marean y Kim, 1998; Marean et al., 1992; Scott y Klein, 1984) han confirmado la predilección de estos por las zonas esponjosas de los huesos largos, debido a su alto nivel nutritivo en comparación con los fragmentos de diáfisis. Carnívoros como los cánidos suelen fracturar los huesos llevando a cabo una intervención intensa sobre las epífisis proximales de los huesos largos con la intención de crear una fisura en el eje del mismo (Binford, 1981; Haynes, 1983). En todo caso, se debe tener en cuenta que las interpretaciones se hacen sobre un conjunto caracterizado como palimpsesto, y por tanto es posible que exista más de una combinación de acciones sobre las epífisis que desemboquen en su práctica desaparición.

\subsection{La fracturación ósea}

El Nivel 4 presenta una elevada fragmentación en todas las tallas de peso, en lo que parece ser un aprovechamiento intensivo de las carcasas animales. Existe cierta de sistematización en la localización de algunos impactos de percusión sobre zonas y partes esqueléticas concretas. En el caso de los metacarpos observamos que la totalidad de los impactos se sitúan en la cara anterior, mientras que en los húmeros lo hacen en la posterior y la exterior. La proporción tan similar de delineaciones curvas, longitudinales y transversales no nos permite afirmar el estado de los huesos en el momento de su rotura. Donde sí observamos un predominio claro es en el ángulo del borde de fractura. La inmensa mayoría de los huesos analizados muestran ángulos oblicuos, que nos lleva a sugerir la fracturación en fresco como la principal en el conjunto (Villa y Mahieu, 1991). Tanto el predominio de ángulos oblicuos como la relativa paridad entre las delineaciones es algo que se extiende a todas las tallas identificadas. Del mismo modo, la presencia casi anecdótica de bordes de fractura irregulares y el predominio casi exclusivo de aquellos de carácter suave, tanto en el ámbito general como en el referente a las tallas, nos viene a confirmar lo mencionado anteriormente, que los huesos fueron fracturados mayoritariamente en fresco. Solo una minoría, que presenta bordes irregulares, pudo haber sido fragmentado en estado seco como resultado de otros procesos como el trampling o la presión de sedimentos.

Para realizar un análisis comparativo con el objetivo de comprobar si existe alguna relación entre las distintas características morfológicas de las tallas estudiadas, se decidió relacionar el tipo de talla con las siguientes variables: ángulo, delineación y superficie del paño de fractura.

Se emplearon dos modelos estadísticos, la $\mathrm{R}$ de Pearson y Lambda (Fig. A.1). El primero, midiendo en una escala del 0 al 1 en valores absolutos determina si hay una correlación perfecta entre variables, y el segundo, tomando los mismos valores, determina la independencia o la dependencia de unas variables. Mediante Lambda y la R de Pearson se calculó el grado de asociación entre las tallas y las tres variables a comparar. Según Lambda, sabiendo la talla no podemos predecir el resto de variables y viceversa. Esto quiere decir que al obtener valores cercanos a 0 , no existe dependencia entre ellas (ver tabla de contingencia en anexo). Según la R de Pearson, no es lo mismo obtener un valor u otro en función de la talla, pero sí existe una correlación no lineal (el valor del coeficiente es cercano a 0) aunque no permite predecir, es decir, no hay grado de dependencia ya que la variable talla y las variables ángulo, delineación y superficie crecen de forma distinta. Por tanto, podemos afirmar que ambos test estadísticos concluyen que son variables independientes. Por tanto, podemos afirmar que ambos test estadísticos concluyen que son variables independientes.

La elevada fragmentación de los huesos que hemos observado, tanto en la longitud como en la sección o circunferencia diafisaria que conservan, nos evidencia que la fractura de los huesos encuentra su origen en la acción antropogénica. La diferenciación antrópico-carnívora, basada en la sección de circunferencia de la diáfisis enunciada por Bunn (1983) y Capaldo y Blumenschine (1994), establece que bajo la acción de agentes animales suele encontrarse la sección completa o semi-completa, mientras que tras el procesado humano la longitud de dicha superficie no supera el 50\%. Recientemente se ha publicado un estudio en el que a través de machine learning algorithms se ha realizado 
una correcta clasificación entre conjuntos fracturados antrópicamente, y otros generados por carnívoros como hienas o lobos (Moclán et al., 2019). En Prado Vargas las muescas de percusión documentadas presentan ángulos cóncavos o convexos, bordes redondeados y puntos de impacto ovales o circulares, elementos diagnósticos de fracturación intencional atribuidos a la acción antrópica (Blumenschine y Selvaggio, 1988, 1991; Capaldo y Blumenschine, 1994; Johnson, 1985; Lyman, 1987; Yravedra, 2006b). Así mismo reseñar que se han encontrado dos restos con evidencias de peeling incipiente, ambos en restos de tallas pequeñas y pertenecientes a porciones de metáfisis. (PV'17 G29/119 y PV'17 G29/291). La disposición de muescas de forma consecutiva (18,39\%), correlativa (6,90\%) o superpuesta $(1,72 \%)$ nos indica, además de un elevado grado de procesado, que la percusión realizada en determinadas regiones del hueso fue reiterada en ciertas ocasiones, llegándose a encontrar 6 impactos en un mismo fragmento. La existencia muescas opuestas nos podría hablar del empleo de un yunque, cuyo uso se vería corroborado por el hallazgo de uno de ellos en la campaña de excavación de 2016.

La extracción de médula se documenta en todas las tallas y elementos esqueléticos predominantes. Tanto en tallas medias como pequeñas, los impactos se sitúan mayoritariamente en la diáfisis. Observamos la tendencia general que enuncian Olsen y Shipman (1988), la cual nos indica que los homínidos percutieron en la zona más frágil del hueso para fracturarlo. En cuanto a las caras en las que se sitúan los impactos en función del elemento esquelético, destacamos que en el húmero prima la posterior y la exterior; en la tibia, los hallados en la interior y la posterior; y en el fémur y los metápodos, los documentados en la anterior. Esto podría tener relación con un patrón conductual; sin embargo, no se observan una repetición estadísticamente significativa como para apoyar esta hipótesis, dado que en términos generales observamos una presencia de impactos en diversas caras. Sólo observamos quizá un conato de especialización o reiteración en los metacarpos y los húmeros. Comparando con los datos ofrecidos por Patou-Mathis (1993) y Blasco et al. (2013), obtenemos algunas concordancias con el último estudio citado, concretamente con los subniveles XVIla y XVIIc de la Cova del Bolomor.

Tal y como recoge Cáceres (2002), para obtener la grasa medular de un modo más sencillo, los huesos se podrían exponer a una fuente de calor. Ésta además de modificar las características físicas del hueso, licua la médula. Es por ello que la exposición ha de ser controlada y limitada. De este modo se obtendrían resultados que coinciden con los nuestros, una presencia relativamente notable de ángulos mixtos y el predominio del grado $0(94,29 \%)$ o de la ausencia de rastros de cremación. Sin embargo, el predominio del mencionado grado de cremación no significa que no se hayan expuesto a un foco de calor, sino que arqueológicamente es más difícil de reconocer. De este modo, los huesos de nuestro estudio pudieron haber sido calentados, pero no haber dejado un cambio de coloración que se pueda observar a simple vista.

\subsection{El papel de otros agentes tafonómicos no antrópicos}

La presencia de mordeduras de carnívoros nos indica que también estos animales tuvieron acceso a los restos que componen el conjunto probablemente cuando los humanos no se encontraban en el yacimiento y aprovechando los desperdicios de estos y/o introduciendo algunos elementos esqueléticos. No obstante, si la acumulación hubiera sido de carnívoros encontraríamos un perfil esquelético ecléctico (Bunn y Pickering, 2010 y Domínguez-Rodrigo et al., 2014). Esto contrasta con los datos obtenidos en nuestro estudio, donde la inmensa mayoría son individuos adultos.

En cuanto a las alteraciones fosildiagénéticas, las agrietaciones, descamaciones y exfoliaciones, como recoge Behrensmeyer (1978), se producen por cambios de temperatura y humedad. Éstas pueden fisurar e incluso ser causantes de la fragmentación del hueso. Sin embargo, como cita la mencionada autora y Haynes (1983), en ambientes húmedos, es decir, en las cuevas, el weathering no es tan rápido ni tan frecuente, por lo que la presencia de éste podría también deberse a la intrusión de elementos en el yacimiento. La presencia de concreción varía también según la humedad, especialmente con los cambios de ésta en el substrato que dan lugar a la dilatación y compactación del sedimento, así como a una cimentación parcial o total de la matriz sedimentaria (Courty et al. 1989). Sin embargo, el porcentaje de concreción en los restos es mínimo. La humedad también se manifiesta en forma de manganesos. La gran cantidad de ellos nos habla de la presencia de charcos o de corrientes de agua de mayor o menor intensidad (Coard, 1999; Fernández-Jalvo y Andrews, 2000). Esto podría estar relacionado con un cierto grado de abrasión. Según Shipman y Rose (1983) la abrasión encuentra su origen en agentes eólicos e hídricos y se diferencian en el número de caras a las que afectan. Sobre esta base, la abrasión de los restos estudiados en Prado Vargas encuentra su origen en la acción del agua, ya que los redondeamientos y pulidos se encuentran en las dos caras del hueso.

Tanto la abrasión como la presión sedimentaria dependen de la matriz sedimentaria, tanto en tipo como en grado (Bromage, 1984; Shipman, 1981). A pesar de la composición sedimentológica del Nivel 4, predominantemente arcillosa, no se ha encontrado ningún cambio en la morfología original de los huesos analizados (Shipman, 1981). Sin embargo, se debe tener en cuenta que el proceso de fosilización trae consigo el paso del hueso a un estado seco, aumentando su fragilidad y facilitando su fragmentación. Éste podría ser el origen de aquellos paños de fractura minoritarios que sugieren 
procesos diagenéticos, fundamentalmente de aquellos con superficie irregular. El trampling también se ve condicionado por la matriz sedimentaria. Olsen y Shipman (1988) indican que está asociado al tránsito biológico, ya sea de homínidos como de animales, traduciéndose en cambios físicos como la ruptura o fragmentación, dependiendo ésta fundamentalmente del sedimento y del enterramiento. Por tanto, habrá mayor probabilidad de que el resto se fragmente cuanto más afectado por el weathering se encuentre. Sin embargo, no descartan que el hueso en estado fresco pueda verse fragmentado por el trampling, aunque lo relega a un plano más minoritario. La presencia de muescas en el borde de fractura (Blasco et al., 2008) asociadas al pisoteo, se sitúan especialmente en paños de fractura oblicuos, un aspecto que concuerda con los datos obtenidos en nuestro estudio. Si bien es cierto, que las muescas que hemos documentado nosotros son un mínimo porcentaje de las marcas de trampling observadas.

Por último, las vermiculaciones $(N R=442)$ y la corrosión química $(N R=442)$ no han mostrado una gran presencia (Tabla A.1). No obstante, éstas nos atestiguan la posición cercana de los restos al exterior, es decir, a la entrada de luz. Estas podrían explicar en cierto modo el porcentaje de weathering identificado $(24,96 \%)$ (Tabla A.1), dado que los huesos podrían verse afectados no solo la exposición solar, sino a otros elementos climatológicos que causaran tal alteración.

\section{CONCLUSIONES}

El conjunto faunístico procedente del Nivel 4 de la Cueva de Prado Vargas evidencia un tipo de hábitat ocupado por grupos neandertales durante el Pleistoceno superior. A partir de los datos obtenidos, se observa una direccionalidad hacia taxones de talla media y pequeña característicos de ambientes abiertos (Cervus elaphus y Equus ferus) y de espacios de montaña ( $R u$ picapra pyrenaica y Capra pyrenaica). El acceso de los homínidos a las presas podría considerarse primario, realizando un primer procesamiento en el punto de obtención y transportando a la cavidad los elementos con mayor contenido medular (estilopodios, zeugopodios y metapodios) sin discriminar la talla y peso del animal.

La presencia de carnívoros en el depósito es esporádica, acudiendo en momentos de ausencia humana. Estos predadores aprovecharían los despojos abandonados por los neandertales e incluso podrían introducir alguna presa o porción anatómica para su consumo usando la cueva como refugio temporal.

Los restos presentan un elevado grado de fracturación, como observamos en la longitud y en la sección diafisaria. Podemos establecer que los homínidos fracturaron los huesos en estado fresco con el objetivo de acceder a la grasa medular. Podemos decir, además, que los huesos fueron fracturados mediante percusión directa, algo corroborado por la presencia de muescas opuestas o contragolpes, que nos indican el empleo de un yunque. Aunque en la inmensa mayoría de los casos se percute en la diáfisis, no se observan unos patrones claros en la selección del punto de impacto, por lo que podemos decir que no parece haber una tradición cultural en este aspecto.

Las bajas proporciones de weathering y de concreción indican que las condiciones de humedad y temperatura en la cueva no fueron tan constantes como para dejar un alto impacto en el registro. Su poco efecto determina la baja efectividad del trampling y la presión de sedimentos. Los manganesos, los pulidos y los redondeos nos indican también de la presencia de corrientes de agua de poca intensidad. La baja presencia de vermiculaciones y corrosión química termina por confirmar que los procesos fosildiagenéticos tuvieron poca incidencia en la fragmentación de los huesos.

\section{AGRADECIMIENTOS}

Este estudio recibe el apoyo de la Junta de Castilla y León desde la Consejería de Cultura y Turismo, Dirección General de Patrimonio Cultural, financiando el proyecto "Intervención arqueológica en la Cueva de Prado Vargas, en Cornejo (Merindad de Sotoscueva, Burgos) con número de expediente B2018/003394. Así mismo agradecer el apoyo incondicional de Beni, el dueño del prado, y de los alcaldes de la Merindad de Sotoscueva y Cornejo. Este trabajo no hubiera podido realizarse sin todos aquellos que han participado y participan en las campañas de excavación, especialmente a los dos codirectores que faltan por mentar en este artículo, Rodrigo Alonso y Alfonso Benito-Calvo, y a quienes componen el núcleo del Laboratorio de Prehistoria, Marta Santamaría, Claudia Santamaría y Pedro Alonso.

\section{BIBLIOGRAFÍA}

Agenbroad, L.D., 1989. Spiral Fractured mammoth Bone from Nonhuman Taphonomic Processes at Hot Spings Mammoth Site. In: Bonnichsen, R. and Sorg, M. H. (ed.), Bone Modification, 139-148. University of Maine Center for the Study of the First Americans, Orono.

Altuna, J., 1971. Los mamíferos del yacimientoprehistórico de Morín. In: González Echegaray, J., Freeman, L.G. (Eds), Cueva Morín: Excavaciones 1966-1968, 367-398. Patronato de las Cuevas Prehistóricas, Santander:

Altuna, J., 1973. Fauna de mamíferos de la Cueva de Morín. In: González Echegaray, J., Freeman, L.G. (Eds), Cueva Morín: Excavaciones 1969, 281-290. Patronato de las Cuevas Prehistóricas; Santander.

Álvarez, M.T., Morales, A., Sesé, C., 1992. Mamíferos DEL yacimiento del Pleistoceno superior de Cueva Millan (Burgos, España). Estudios geológicos 48, 193-204.

Andrews, P., Jalvo, Y.F., 1997. Surface modifications of the Sima de los Huesos fossil humans. Journal of Human Evolution 33, 191-217.

Arceredillo, D., 2015. Análisis Paleobiológico de los ungulados del Pleistoceno Superior de la Meseta Norte. Universidad de 
Salamanca, Facultad de Ciencias, departamento de Geología. Tesis doctoral.

Arilla, M., Rosell, J., Blasco, R., Domínguez-Rodrigo, M., Pickering, T.R., 2014. The "Bear" essentials: actualistic research on Ursus arctos arctos in the Spanish Pyrenees and its implications for paleontology and archaeology. PLoS ONE 9, e102457.

Arriaza, M.C., Yravedra, J., Domínguez-Rodrigo, M., Maté-González, M.A., García Vargas, E., Palomeque-González, J.F., Aramendi, J., González-Aguilera, D., Baquedado, E., 2017. On applications of micro-photogrammetry and geometric morphometrics to studies of tooth mark morphology: the modern Olduvai carnivore site (Tanzania). Palaeogeogr. Palaeoclimatol. Palaeoecol. 488, 103-112.

Arriaza, M.C., Aramendi, J., Maté-González, M.A., Yravedra, J., Baquedano, E., González-Aguilera, D., Domínguez-Rodrigo, M., 2019. Geometric-morphometric analysis of tooth pits and the identification of felid and hyenid agency in bone modification. Quaternary International 517, 79-87.

Azorit, C., Analla, M., Carrasco, R., Calvo, J.A., Múñoz Cobo, J., 2002. Teeth eruption pattern in red deer (Cervus elaphus hispanicus) in southern Spain. Anales de Biología 24, 107-114.

Baldeón, A., 1999. El abrigo de Axlor (Bizkaia, País Vasco). Las industrias líticas de sus niveles Musterienses. Munibe Antropologia-Arkeologia 51, 9-121.

Bartram, L.E., Marean, C.W., 1999. Explaining the "Klasies Pattern" p2663x2315: Kua Ethnoarchaeology, Die Kelders Middle Stone Archaeofauna, Long Bone Fragmentation and Carnivore Ravaging. Journal of Archaeological Science 26, 9-29.

Behrensmeyer, A.K., 1978. Taphonomic and ecological information from bone weathering. Paleobiology 4, 150-162.

Behrensmeyer, A. K., 1990. Transport-hydrodinamics: bones. In: Briggs, D.E.G., Crowther, P. R. (eds.), Paleobiology: a Synthesis, 232-235. Blackwell Scientific Publications, Oxford.

Binford, L.R., 1978. Nunamiut Ethnoarchaeology. Academic Press, New York.

Binford, L.R., 1981. Bones: Ancient Men and Modern Myths. Academic Press, New York.

Binford, L.R., 1984. Butchery sharing and the archaeological record. Journal of Anthropological Archaeology 3, 235-237.

Binford, L.R., 1988. Fact and fiction about the Zinjanthropus floor: data, arguments, and interpretations. Curr. Anthropol. 29, 123-135.

Binford, L.R., 1989. Étude taphonomique des restes fauniques de la Grotte Vaufrey, Couche VIII. In: Rigaud, J.P., La Grotte Vaufrey: Paléoenvironnement, chronologie, activités humaines. Mémoires de la Société Préhistorique Française 19, 535-564.

Blasco, R., 2006. Estrategias de Subsistencia de los Homínidos del Nivel XII de la Cova del Bolomor (La Valldigna, Valencia), Departamento de Historia, Historia de Arte y Geografía, Universitat Rovira i Virgili, Tarragona.

Blasco, R., 2008. Consumo humano de tortugas en el nivel IV de la cueva de Bolomor (Valencia, España). Journal of Archaeological Science 35, 2839-2848.

Blasco, R., Peris, J. F., 2009. Consumo de aves del Pleistoceno Medio en el Nivel XI de la Cueva de Bolomor (Valencia, España). Diario de la ciencia arqueológica 36(10), 2213-2223.

Blasco, R., Fernández-Peris, J., 2012. A uniquely broad spectrum diet during the Middle Pleistocene at Bolomor Cave (Valencia, Spain). Quaternary International 252, 16-31.
Blasco, R., Rosell, J., Fernández Peris, J., Cáceres, I., Vergès, J. M., 2008. A new element of trampling: an experimental application on the Level XVII faunal record of Bolomor Cave (Valencia, Spain). Journal of Archaeological Science 35, 1605-1618.

Blasco, R., Domínguez-Rodrigo, M., Lozano, S., Pastó, I., Riba, D., Vaquero, M., Fernández Peris, J., Arsuaga, J.M., Bermúdez de Castro, J.M., Carbonell, E., 2013. Learning by Heart: Cultural Patterns in the Faunal Processing Sequence during the Middle Pleistocene. PLoS one 8(2): e55863.

Blumenschine, R.J., 1988. An experimental model of the timing of hominid and carnivore influence on archaeological bone assemblages. Journal of Archaeological Science 15, 483-502.

Blumenschine, R.J., 1991. Hominid carnivory and foraging strategies and the socio-economic function of early archaeological sites. Phil. Trans. R. Soc. Lon. 334, 211-221.

Blumenschine, R.J., 1995. Percussion marks, tooth marks, and experimental determinations of the timing of hominid and carnivore access to long bones at FLK Zinjanthropus, Olduvai Gorge, Tanzania. Journal of Human Evolution 29(1), 21-51.

Blumenschine, R.J., Prassack, K., Kreger, C.D., Pante, M.C., 2007. Carnivore tooth-marks, microbial bioerosion, and the invalidation of Domínguez-Rodrigo and Barba's (2006) test of Oldwoman hominin scavenging behaviour. Journal of Human Evolution 53(4), 420-426.

Blumenschine, R.J., Selvaggio, M.M., 1988. Percussion marks on bone surfaces as a new diagnostic of hominid behaviour. Nature 333, 763-765.

Blumenschine, R.J., Selvaggio, M.M., 1991. Hominid carnivory and foranging strategies, and the socio-economic function of early archaeological sites. Phil. Trans. R. Soc. Lond. B 334, 211-221.

Brain, C.K., 1981. The Hunters or the Hunted? An Introduction to African Cave Taphonomy. University of Chicago Press, Chicago.

Brett, C.E., 1990. Destructive taphonomic processes and skeletal durability. In: Briggs D.E.G., Crowther, P.R. (eds.), Paleobiology, 223-226. Oxford.

Bromage, T.G., 1984. Interpretation of Scanning Electron Microscope Images of Abraded Forming Bone Surfaces. American Journal of Physical Anthropology 64, 161-178.

Bromage, T.G., 1985. Systematic inquiry in tests of negative/ positive replica combinations for SEM. Journal of Microscopy 64(137), 209-216.

Bromage, T.G., Boyde, A., 1984. Microscopic criteria for the determination of directionality cutmarks on bone. American Journal of Physical Anthropology 65, 359-366.

Brown, K., Fa, D.A., Finlayson, G., Finlayosn, C., 2011. Small game and marine resource exploitation by Neanderthals: the evidence from Gibraltar. In: Bicho, N., Haws, J. (Eds.), Trekking the Shore: Changing Coastlines and the Antiquity of Coastal Settlement. Interdisciplinary Contribution to Archaeology, 247-271.Springer, New York.

Bunn, H.T, 1981. Archaeozoological evidence for meat-eating by Plio-pleistocene hominids from Koobi For a and Olduvai Gore. Nature 291, 574-577.

Bunn, H.T., 1983. Comparative Analysis of Modern Bone Assemblages from a San Hunter-Gatherer Camp in the Kalahari Desert, Botswana, and from a Spotted Hyena Den Near Nai- 
robi, Kenia. In Grigson, E. b. J. C.-B. a. C. (ed.) Animals and Archaeology: 1.- Hunters and Their Prey. Oxford, British Archaeological Reports. International Series 163, 143-148.

Bunn, H.T, 1986. Patterns of Skeletal Representation and Hominid Subsitence Activities at Olduvai Gore, Tanzania, and Koobi For a, Kenya. Journal of Human Evolution 15, 673-690.

Bunn, H.T., 1989. Diagnosing Plio-Pleistocene Hominid Activity with Bone Fracture Evidence. In Bonnichsen, R., Sorg, M.H. (ed.) Bone Modification. Orono, University of Maine Center for the Study of the First Americans, 299-315.

Bunn, H.T., Bartram, L.E., Kroll, E.M., 1988. Variability in bone assemblage formation from Hadza hunting, scavenging, and carcass processing. Journal of Anthropological Archaeology 7 (4), 412-457.

Bunn, H.T., Kroll, E.M., 1986. Systematic butchery by Plio/ Pleistocene hominids at Olduvai Gorge, Tanzania [and Comments and Reply]. Curr. Anthrop. 27, 431-452.

Bunn, H.T., Kroll, E.M., 1988. Fact and fiction about the Zinjanthropus floor: data, arguments, and interpretations. Curr. Anthropol. 29, 135-149.

Bunn, H. T., Ezzo, J.A., 1993. Hunting and scavenging by plio-pleistocene hominids: nutritional constraints, archaeological patterns and behavioural implications. Journal of Archaeological Science 20, 365-398.

Bunn, H.T., Pickering, T.R., 2010. Methodological recommendations for ungulate mortality analyses in paleoanthropology. Quatern. Res. 74, 388-394.

Cáceres, I., 1995. Estudios tafonómicos de los procesos de formación del Nivel I del Abric Romaní (Capellades, Barcelona): La influencia de la actividad antrópica. Tesis de licenciatura Dpt. D'Història i Geografia. Universita Rovira i Virgili, Tarragona.

Cáceres, I., 2002. Tafonomía de yacimientos antrópicos en karst. Complejo Halería (Sierra de Atapuerca, Burgos), Vanguard Cave (Gibraltar) y Abric Romaní (Capellades, Barcelona). Tesis doctoral. Dpt. Història I Geografia Rovira i Virgili, Tarragona.

Cáceres, I., Bravo, P., Esteban, M., Expósito, I., Saladié, P., 2002. Fresh and heater bones breakage. An experimental approach. Current topics on taphonomy and fossilization 30, 417-479.

Capaldo, S.D., 1995. Inferring hominid and carnivore behavior from dual-patterned archaeofaunal assemblages. Ph.D. Dissertation, Rutgers University.

Capaldo, S.D., Blumenschine, R.J., 1994. A Quantitative Diagnosis of Notches Made by Hammerstone Percussion and Carnivore Gnawing on Bovid Long Bones. American Antiquity 59, 724-748.

Castaños, P.M., 2005. Revisión actualizada de las faunas de macromamiferos del Würm antiguo en la Región Cantábrica. Actas de la reunión científica "Neandertales cantábricos, estado de la cuestión", 201-207.

Chase, P.G., 1986. The hunters of Combe Grenal: approaches to Middle Paleolithic subsistence in Europe. British Archaeological Reports International Series. Oxford.

Chase, P.G., 1989. How different was Middle Palaeolithic subsistence? A zooarchaeological perspective on the Middle to Upper Palaeolithic transition. In: Mellars, P.A., Stringer, C.B. (Eds.), The Human Revolution: Behavioral and Biological Perspectives on the Origins of Modern Humans, 321-327. Edinburgh University Press, Edinburgh.
Coard, R., 1999. One bone, two bones, wet bones, dry bones: transport potentials under experimental conditions. Journal of Archaeological Science 26, 1369-1375.

Conard, N.J., Prindiville, T.J., 2000. Middle Palaeolithic Hunting Economies in the Rhineland. International Journal of Osteoarchaeology 10, 286-309.

Costamagno, S., 2013. Bone Grease Rendering in Mousterian Contexts: The Case of Noisetier Cave (Fréchet-Aure, Hautes-Pyréneés, France). Zooarchaeology and Modern Human Origins: Human Hunting Behavior during the Later Pleistocene, Vertebrate Paleobiology and Paleoanthropology.

Costamagno, S., Théry-Parisot, I., Brugal, J.P., Guilbert, R., 2005. Taphonomic consequences of the use of bones as fuel. Experimental data and archaeological applications. In: O'Connor, T. (Ed.), Biosphere to Lithosphere, New Studies in Vertebrate Taphonomy, (Proceed. 9th Conf. ICAZ, Durham, Aug. 02), 51-62.. Oxbow Books, Durham.

Costamagno, S., Meignen, L., Beauval, C., Vandermeersch, B., Maureille, B., 2006. Les Pradelles (Marillac-le-Franc, France): a mousterian reindeer hunting camp? Journal of Anthropological Archaeology 25(4), 466-484.

Courty, M.A., Goldberg, P., MaCphail, R., 1989. Soils and micromorphology in archaeology. Cambridge University Press, Cambridge.

Daujeard, C., Moncel, M.-H., 2010. On Neanderthal subsistence strategiesand land use: a regional focus on the Rhone Valley area in southeastern France. Journal Anthropological Archaeology 9, 368-391.

Delaney-Rivera, C., Plummer, T.W., Hodgson, J.A., Forrest, F., Hertel, F., Oliver, J.S., 2009. Pits and pitfalls: taxonomic variability and patterning in tooth mark dimensions. Journal of Archaeological Science 36, 2597-2608.

Delpech, F., Villa, P., 1993. Activités de chasse et de boucherie dans la Grotte des Églises. In: Desse, J., Audoin-Rouzeau, F. (éds), Exploitation des animaux sauvages à travers le temps: XIIle rencontres internationales d'archéologie et d'histoire d'Antibes - IVe colloque international de l'Homme et l'Animal, Société de recherche interdisciplinaire, Antibes, 15-17 octobre 1992. Anthropozoologica HS (1), 79-102.

Díez, J. C., Fernández Jalvo, Y., Cáceres, I., Rosell, J., 1999. Zooarchaeology and Taphonomy of Aurora Stratum (Gran Dolina, Sierra de Atapuerca, Spain). Journal of Human Evolution 37, 623-657.

Díez, C., Alonso, R., Bengoechea, A., Colina, A., Jordá, J.F., Navazo, M., Ortiz, J.E., Pérez, S., Torres, T., 2008. El paleolítico medio en el valle del Arlanza (Burgos). Los sitios de la Ermita, Millán y la Mina. Rev. C. \& G., 22(3-4), 135-157.

Domínguez-Rodrigo, M., Piqueras, A., 2003. The use of tooth pits to identify carnivore taxa in toothmarked archaeofaunas and their relevance to reconstruct hominid carcass processing behaviours. Journal of Archaeological Science 30, 1385-1391.

Domínguez-Rodrigo, M., Barba, R., 2006. New estimates of tooth mark and percussion mark frequencies at the FLK Zinj site: the carnivore-hominid-carnivore hypothesis falsified. J. Hum. Evol. 50, 170-194.

Domínguez-Rodrigo, M., Baquedano, E., 2018. Distinguishing butchery cut marks from crocodile bite marks through machine learning methods. Sci. Rep. 8(1), 5786.

Domínguez-Rodrigo, M., de Juana, S., Galán, A.B., Rodríguez, M., 2009. A new protocol to differentiate trampling marks from butchery cut marks. J. Archaeol. Sci. 36, 2643-2654. 
Domínguez-Rodrigo, M., Bunn, H., Yravedra, J., 2014. A critical re-evaluation of bone surface modification models for inferring fossil hominin and carnivore interactions through a multivariate approach: application to the FLK Zinj archaeofaunal assemblage (Olduvai Gorge, Tanzania). Quatern. Int. 322, 32-43.

Domínguez-Rodrigo, M., Saladié, P., Cáceres, I., Huguet, R., Yravedra, J., Rodríguez-Hidalgo, A., Martín, P., Pineda, A., Marín, J., Gené, C., Aramendi, J., Cobo-Sánchez, L., 2017. Use and abuse of cut marks analyses: The Rorschach effect. Journal of Archaeological Science 86, 14-23.

Egeland, C.P., Pickering, T.R., Domínguez-Rodrigo, M., Brain, C.K., 2004. Disentangling Early Stone Age palimpsests: determining the functional independence of hominid- and carnivore-derived portions of archaeofaunas. J. Hum. Evol. 47, 343-357.

Eixea, A., Villaverde, V., Zilhão, J., Bergadà, M., Sanchis, A., Morales, J.V., Real, C., Martínez, J.A., 2012. Variation in the use of space through time at Abrigo de la Quebrada (Chelva, Valencia): the case of Middle Paleolithic levels IV and VII. In: García, A., García, J., Maximiano, A., Ríos-Garaizar, J. (Eds.), Debating Spatial Archaeology. Proceedings of the International Workshop on Landscape and Spatial Analysis in Archaeology, 153-166. Santander

Emerson, A.M., 1993. The role of Body Part Utility in Small-scale Hunting under Two Strategies of Carcass Recovery El papel de la Utilidad de las Partes Anatómicas en la Caza a Pequeña Escala bajo Dos Estrategias de Explotación de las Carcasas 1-17.

Enloe, J., 1991. Subsistence organization in the Upper Palaeolithic: carcass refitting and food sharing at Pincevent. PhD Dissertation, University of New Mexico.

Fernández-Jalvo, Y., 1992. Tafonomía de Microvertebrados del Complejo Kárstico de Atapuerca (Burgos). Tesis Doctoral, Departamento de Paleontología, Universidad Complutense de Madrid.

Fernández-Jalvo, Y., 1999. Tafonomía de micromamíferos del yacimiento Galería de Atapuerca (Burgos). In: Carbonell, E., Rosas, A., Díez, J.C. (ed.) Atapuerca: Ocupaciones Humanas y Paleoecología del Yacimiento de Galería. Zamora, Junta de Castilla y León. Consejería de Educación y Cultura. Memorias 7, 95-128.

Fernández-Jalvo, Y., Andrews, P., 2000. The taphonomy os pleistocene cavez, with particular reference to Gibraltar. In: Stringer, C.B., Barton, R.N.E., Finlayson, J.C. (eds.), Neanderthals on the Edge, 171-182. Oxbow Books, Oxford.

Fernández-Jalvo, Y., Andrews, P., 2016. Atlas of Taphonomic Identifications. Springer.

Fiorillo, A.R., 1989. An experimental study of trampling: implications for the fossil record. In: Bonnichsen, R., Sorg, M. (Eds.), Bone Modification, 61-71. Center for the Study of First Americans, Orono, Maine.

Fisher, J.W., 1995. Bone Surface Modifications in Zooarchaeology. Journal os Archaeological Method and Theory 2(1), 7-68.

Fuentes Vidarte, C., 1980. Estudio de la fauna de "El Pendo". En J. González Echegaray (Ed.), El Yacimiento de la Cueva de "El Pendo".Madrid: Bibliotheca Praehistorica Hispana 17, 215-237.

Gabucio, M.J., Cáceres, I., Rosell, J., Saladié, P., Vallverdú, J., 2014. From small bone fragments to Neanderthal activity areas: the case of Level $O$ of the Abric Romaní (Capellades, Barcelona, Spain). Quat. Int. 330, 36-51.

Gabucio, M.J., Fernández-Laso, M.C., Rosell, J., 2017. Turning a rock shelter into a home. Neanderthal use of space in Abric Romaní levels M and O. Hist. Biol. 1-24.
Gaudzinski, S., 2006. Monospecific or species-dominated faunal assemblages during the middle palaeolithic in Europe. In: Hovers, E., Kuhn, S. (Eds.), Transitions Before the Transition. Evolution and Stability in the Middle Palaeolithic and Middle Stone Age, 137e147. Springer, New York.

Gaudzinski, S., Roebroeks, W., 2000. Adults only. Reindeer hunting at the Middle Palaeolithic site Salzgitter Lebenstedt, Northern Germany. Journal of Human Evolution 38, 497-521.

Gaudzinski-Windheuser, S., Niven, L., 2009. Hominid subsistence patterns during the Middle and Late Paleolithic in Northwestern Europe. In: Hublin, J.-J., Richards, M.P. (Eds.), The Evolution of Hominin diets, 99-111. Springer, Dordrecht.

Gifford-Gonzalez, D.P., 1989. Ethnographic analogues for interpreting modified bones: some case from East Africa. In: Bonnichsen, R., Sorg, M.H. (ed.), Bone Modification, 179-246. University of Maine Center for the Study of the First Americans, Orono.

González, J., Ibañez, J.J., Ríos, J., Bourguignon, L., Castaños, P., Tarriño, A., 2005. Excavaciones recientes en Axlor. Movilidad y planificación de actividades en grupos de neandertales. Monografías del Museo de Altamira 20, 527-539.

Grayson, D., Delpech, F., 2003. Ungulates and the Middle-to-Upper Paleolithic transition at Grotte XVI (Dordogne, France). Journal of Archaeological Science 30, 1633-1648.

Haynes, G., 1980. Evidence of carnivore gnawing on Pleistocene and Recent mamalian bones. Paleobiology 6, 341-351.

Haynes, G., 1991. Mammoths, Mastodonts, and Elephants: Biology, Behavior, and the Fossil Record. Cambridge University Press, Cambridge.

Haynes, G., 1983. Frequencies of Spiral and Green-Bone Fractures on Ungulate Limb Bones in Modern Surface Assemblages. American Antiquity 48, 102-114.

Horwitz, K.L., 1990. The origin of partially digested bones recovered from archaeological contexts in Israel. Paléoríent 16, 97-106.

Irving, W. N., Jopling, A. V., Kritsch-Amstrong, I., 1989. Studies of Bone Technology and Taphonomy, Old Crow Basin, Yukon Territory. In: Bonnichsen, R., Sorg, M.H. (ed.) Bone Modification, 347 -379. University of Maine Center for the Study of the First Americans, Orono.

Johnson, E., 1985. Current developments in bone technology. Adv. Archaeol.Method Theory 8, 157-235.

Karr, L., 2015. Human use and reuse of megafaunal bones in North America: bone fracture, taphonomy, and archaeological interpretation. Quat. Int. 361, 332-341.

Karr, L.P., Outram, A.K., 2012. Tracking changes in bone fracture morphology over time: environment, taphonomy, and the archaeological record. J. Archaeol. Sci. 39(2), 555-559.

Kindler, L., Smith, G.M., Wagner, M., 2014. Introduction to faunal analysis at NeumarkNord 2. In: Gaudzinski-Windheuser, S., Roebroeks, W. (Eds.), Multidisciplinary Studies of the Middle Palaeolithic Record from Neumark-Nord (Germany) vol. 1, 197211. Landesmuseum Für Vorgeschichte, Halle. Band 69.

Klein, R.G., Cruz-Uribe, K., 1984. The analysis of animal bones from archeological sites. Chicago University Press, Chicago \& London.

Lam, Y.M., Chen, X., Pearson, O.M., 1999. Intertaxonomic Variability in Patterns of Bone Density and the Differential Repre- 
sentation of Bovid, Cervid, and Equid Elements in the Archaeological Record. American Antiquity 64(2), 343-362.

Luret, M., Burke, A., Bernaldo de Quiros, F., Besse, M., 2020. El Castillo cave (Cantabria, Spain): Archeozoological comparison between the Mousterian occupation level (unit 20) and the "Aurignacien de transition de type El Castillo" (unit 18). Journal of Archaeological Science. Reports 31, 102339.

Lyman, R.L., 1985. Bone frecuencies: differential survivorship of fossil classes. Journal of Archaeological Science 12, 221-236.

Lyman, R.L., 1987. Archaeofaunas and butchery studies: a taphonomic perspective. Advances in Archaeological Method and Theory 10, 249-337.

Lyman, R.L., 1994. Vertebrate Taphonomy. Cambridge University Press, Cambridge.

Lyman, R.L., 2008. Estimating the magnitude of data asymmetry in palaeozoological biogeography. International Journal of Osteoarchaeology 18, 85-94.

Maguire, J. M., Pemberton, D., Collett, M. H., 1980. The Makapansgat Limeworks grey breccia: Hominids, hyaenas, hystricids or hillwash? Paleontologia Africana 23, 75-98.

Marean, C.W., Kim, S.Y., 1998. Mousterian Large-Mammal Remains from Kobeh Cave Behavioral Implications for NeanderthaIs and Early Modern Humans. Current Anthropology 39, 79-114.

Marean, C.W., Spencer, L.M., Blumenschine, R.J., Capaldo, S.D., 1992. Captive hyaena bone choice and destruction, the Schlepp effect and olduvai archaeofaunas. Journal of Archaeological Science 19, 101-121.

Merritt, S.P., Davis, K.M., 2017. Diagnostic properties of hammerstone-broken long bone fragments, specimen identifiability, and Early Stone Age butchered assemblage interpretation. Journal of Archaeological Science 85, 114-123.

Moclán, A., Domínguez-Rodrigo, M., 2018. An experimental study of the patterned nature of anthropogenic bone breakage and its impact on bone surface modification frequencies. Journal of Archaeological Science 96, 1-13.

Moclan, A, Domínguez-Rodrigo, M., Yravedra, J., 2019. Classifying agency in bone breakage: an experimental analysis of fracture planes to differentiate between hominin and carnivore dynamic and static loading using machine learning $(\mathrm{ML})$ algorithms. Archaeological and Anthropological Sciences 11, 4663-4680.

Morin, E., 2010. Taphonomic implications of the use of bone as fuel. In: Théry-Parisot, I., Chabal, L., Costamagno, S. (ed.), The Taphonomy of Burned OrganicResidues and Combustion Features in Archaeological Contexts. P@lethnologie 2, 209-217.

Morlan, R.E., 1980. Taphonomy and archaeology in the Upper Pleistocene of the northern Yukon Territory: a glimpse of the peopling of the New World. 94 Mercury Series, Ottawa.

Morlan, R.E., 1984. Towards the definition of criteria for the recognition of artificial bone alterations. Quat. Res. 22, 160-171.

Navazo Ruiz, M., Díez Fernández-Lomana, J.C., 2008. Prado Vargas y la variabilidad tecnológica a finales del Paleolítico Medio en la meseta norte. Variabilidad técnica en el Paleolítico Medio en el sudoeste de Europa. Treballs d'Arqueologia 14, 121-139, https://www.raco.cat/index.php/TreballsArqueologia/ article/view/148737 121-139.

Navazo Ruiz, M., Díez Fernández-Lomana, J.C., Torres, T., Colina, A., Ortiz, J.E., 2005. La Cueva de Prado Vargas: Un yacimiento del Paleolítico Medio en el Sur de la Cordillera Cantábri- ca. Actas de la Reunión Científica "Neandertales cantábricos, estado de la cuestión", 151-166.

Niven, L., Steele, T.E., Rendu, W., Mallye, J.-B., McPherron, S.P., Soressi,M., Jaubert, J., Hublin, J.-J., 2012. Neandertal mobility and large-gamehunting: the exploitation of reindeer during the Quina Mousterianat Chez-Pinaud Jonzac (Charente-Maritime, France). Journal of Human Evolution 63, 624-635.

O'Connell, J, Hawkes, K, Blurton Jones., N. 1988. Hazda scavenging: implications for Plio-Pleistocene hominid subsistence. Current Anthropology 29(2), 356-363.

Olsen, S.L., Shipman, P., 1988. Surface Modification on Bone: Trampling versus Butchery. Journal of Archaeological Science 15, 535-553.

Outram, A.K., 2001. A new approach to identifying bone marrow and grease exploitation: why the "indeterminate" fragments should not be ignored. Journal of Archaeological Science 28, 401-410.

Outram, A.K., 2002. Bone Fracture and Within-bone Nutrients: An Experimentally Based Method for Investigating Levels of Marrow Extraction. In: Miracle, P., Milner, N. (eds.), 2004. Consuming Passions and Patterns of Consumption, 51-64. McDonald Institute for Archaeological Research, Cambridge.

Patou-Mathis, M.E., 1993. Étude de la fracturation des os longs de renne: Methode et resultats le niveau Magdalenien Archaique de la Grotte de Tournall A Bize (Aude). In: Hannus, L.A., Rossum, L., Winham, R.P. (eds.). Proceedings of the 1993 Bone Modification Conference, Hot Springs, South Dakota. Occasional Publication 1, 195-219. Archaeological Laboratory, Augusta College, Sioux Falls, S. D.

Perlès, C., Masset, C., 1977. Préhistoire du feu [compte-rendu]. L'Homme 17(4), 126-127. Masson ed., Paris.

Pickering, T. R., Egeland, C. P., 2006. Experimental patterns of hammerstone percussion damage on bones: implications for inferences of carcass processing by humans. Journal of $\mathrm{Hu}-$ man Evolution 33, 459-469.

Pickering, T.R., 2001. Taphonomy of the Swartkrans hominid postcrania and its bearing on issues of meat-eating and fire management. In: Stanford, C.B., Bunn, H. (Eds.), Meat-eating and Human Evolution, 33-51. Oxford University Press, New York.

Pike-Tay, A., Cabrera Valdés, V., Bernaldo de Quirós, F., 1999. Seasonal variations of the middle-upper paleolithic transition at El castillo, Cueva Morín and El pendo (Cantabria, Spain). Journal of Human Evolution 36(3), 283-317.

Potapov, L.P., Levin, M.G., 1964. The Peoples of Siberia. University of Chicago Press.

Potts, R., Shipman, P., 1981. Cutmarks made by stone tools on bones from Olduvai Gorge, Tanzania. Nature 291, 577-580.

Rendu, W., 2010. Hunting behavior and Neanderthal adaptability inthe Late Pleistocene site of Pech-de-l'Azé I. Journal of Archaeological Science 37(8), 1798-1810.

Rendu, W., Costamagno, S., Meignen, L., Soulier, M.-C., 2012. Monospecific faunal spectra in Mousterian contexts: implications for social behaviour. Quaternary International 247, 50-58.

Richards, M.P., Trinkaus, E., 2009. Isotopic evidence for the diets of European Neanderthals and early modern humans. Proceedings of the National Academy of Sciences 106, 16034-16039.

Romandini, M., Nannini, N., Tagliacozzo, A., Peresani, M., 2014. The ungulate assemblage from layer A9 at Grotta di Fumane, Italy: a zooarchaeological contribution to the reconstruction of Neanderthal ecology. Quaternary International 337, 11-27. 
Rosell, J., Blasco, R., 2009. Home Sharing: Carnivores in Anthropogenic Assemblages of the Middle Pleistocene. Journal of taphonomy 7(4), 305-324.

Rosell, J., Blasco, R., Huguet, R., Cáceres, I., Saladié, P., Rivals, F., Bennàsar, M., Bravo, P., Campeny, G., Esteban-Nadal, M., Fernández-Laso, C., Gabucio, M.J., Ibáñez, N., Martín, P., Muñoz, L., Rodríguez-Hidalgo, A., 2012. Occupational patterns and subsistence strategies in level j of abric Romaní. In: Carbonell I Roura, E. (Ed.), High Resolution Archaeology and Neanderthal Behavior Time and Space in Level J of Abric Romaní (Capellades, Spain), 313-372. Springer, Dordretch.

Salazar-García D.C., Power, R.C., Sanchis Serra, A., Villaverde V., Walker, M. J., Henry, A. G., 2013. Neanderthal diets in central and southeastern Mediterranean Iberia. Quaternary International 318, 3-18.

Sánchez-Hernández, C., Rivals, F., Blasco, R., Rosell, J., 2014. Short, but repeated Neanderthal visits to Teixoneres Cave (MIS 3, Barcelona, Spain): a combined analysis of tooth microwear patterns and seasonality. J. Archaeol. Sci. 49, 317-325.

Sanchis Serra, A., Fernández Peris, J., 2008. Procesado y consumo antrópico de conejo en la Cova del Bolomor (Tabernas de la Valldigna, Valencia). Complutum, 19(1), 25-46.

Santamaria, C., Luengo, S.M., Navazo, M., Benito-Calvo, A., Martín, I., 2016. Methodological approach and first results of use-wear traces on Flint tools through confocal microscope. DigiAR, 3, 15-22

Schmid, E., 1972. Atlas of Animal Bones, for Prehistorians, Archaeologists, and Quaternary Geologists. Elsevier Publishing Company.

Scott, L., Klein, R.G., 1984. A hyena-accumulated bone assemblage from late Holocene deposits at Deelpan, Orange Free State. Annals of The South African Museum 86, 217-227.

Selvaggio, M.M., 1998. Evidence for a three-stage sequence of hominid and carnivore involvement with long bones at FLK Zinjanthropus, Olduvai Gorge, Tanzania. J. Archaeol. Sci. 25, 191-202.

Selvaggio, M.M., 1994. Evidence from carnivore tooth marks and stone-toolbutchery marks for scavenging by hominids at FLK Zinjanthropus, Olduvai Gorge, Tanzania. Ph.D. Dissertation, Rutgers University.

Selvaggio, M.M., Wilder, J., 2001. Identifying the involvement of multiple carnivore taxa with archaeological bone assemblages. Journal of Archaeological Science 28, 465-470.

Shipman P. 1981. The Life History of a Fossil: An Introduction to Taphonomy and Paleoecology. Harvard University Press, Harvard, Cambridge.

Shipman, P., Rose, J., 1983. Early hominid hunting, butchering, and carcass-processing behaviors: Approaches to the fossil record. Journal of Anthropological Archaeology 2, 57-98.

Shipman, P., Rose, J., 1984. Cutmark Mimics on Modern and Fossil Bovid Bones. Current Anthropology 25, 116-7.

Speth, J.D., 2012. Middle Palaeolithic subsistence in the Near East: Zooarchaeological perspectives - Past, present and future. Before Farming: The Archaeology and Anthropology of HunterGatherers 2(2), 1-45.

Stiner, M.C., 1994, Honor among thieves: A zooarchaeological study of Neandertal ecology. Princeton University Press.

Stiner, M.C., 2002. Carnivory, coevolution and the geographic spread of the genus Homo. Journal of Archaeological Research 10, 1-63.
Stiner, M.C., Kuhn, S.L., Weiner, S., Bar-Yosef, O., 1995. Differential Burning, Recrystallization, and Fragmentation of Archaeological Bone. Journal of Archaeological Science 22, 223-237.

Stiner, M.C., Munro, N.D., Surovell, T.A., 2000. The tortoise and the hare: small-game use, the broad-spectru revolution and Palaeolithic demography. Current Anthropology 41, 39-73.

Tappen, M., Wrangham, R., 2000. Recognizing hominoid-modified bones: the taphonomy of Colobus bones partially digested by free-ranging chimpanzees in the Kibale Forest, Uganda. Am. J. Phys. Anthropol. 113, 217e234.

Tersigni, M.-T.A., 2007. Frozen human bone: a microscopic investigation. J. Forensic Sci. 52(1), 16-20.

Théry-Parisot, I., 2002. Fuel management (bone and wood) during the lower Aurignacian in the Pataud rock shelter (Lower Palaeolithic, Les Eyzies de Tayac, Dordogne, France). Contribution of experimentation. Journal of Archaeological Science 29, 1415-1421.

Théry-parisot, I., Meignen, L., 1998. Économie des combustibles (bois et lignite) dans I' abri moustérien des Canalettes [L'expérimentation à la simulation des besoins énergétiques] L'expérimentation à la simulation des besoins énergétiques. Gallia Préhistoire 42, 45-55.

Villa, P., Mahieu, E., 1991. Breakage pattern of human long bones. Journal of Human Evolution 21, 27-48.

Villaverde, V., Eixea, A., Zilhão, J., Sanchis, A., Real, C., Bergadà, M., 2017. Diachronic variation in the Middle Paleolithic settlement of Abrigo de la Quebrada (Chelva, Spain). Quat. Int. 435 A, 164-179.

White, T.D., 1992. Prehistoric Cannibalism at Mancos 5MTURMR-2346. Princeton University Press.

Yravedra, J., 2006a. Acumulaciones biológicas en yacimientos arqueológicos: Amalda VII y Esquilleu III-IV. Trabajos de Prehistoria 63(2), 55-78.

Yravedra, J., 2006b. Tafonomía aplicada a la zooarqueología. Madrid, UNED. Madrid.

Yravedra, J., Uzquiano, P., 2013. Burnt bone assemblages from El Esquilleu cave (Cantabria, Northern Spain): deliberate use for fuel or systematic disposal of organic waste? Quaternary Science Reviews 68, 175-190.

Yravedra, J., García-Vargas, E., Maté-González, M.A., Aramendi, J., Palomeque-González, J.F., Vallés-Iriso, J., Matesanz-Vicente, J., González-Aguilera, D., Domínguez-Rodríguez, M., 2017a. The use of Micro-Photogrammetry and Geometric Morphometrics for identifying carnivore agency in bone assemblages. Journal os Archaeological Science: Reports 14, 106-115.

Yravedra, J., Maté-González, M.Á., Courtenay, L.A., González-Aguilera, D., Fernández Fernández, M., 2017b. The use of canid tooth marks on bone for the identification of livestock predation. Scientific Reports 9, 16301.

Yravedra Sainz de los Terreros, J., 2004. Interacción de humanos y carnívoros en el Pleistoceno Superior de la Península Ibérica. Novedosas interpretaciones en la cueva de Amalda. Espacio, tiempo y forma. Serie I, Prehistoria y arqueología 79-98.

Yravedra Sainz de Los Terreros, J., Cobo L., 2015. Neanderthal exploitation of ibex andchamois in Souhwetern Europe. J. Human Evolut. 78, 12-32. 
9. ANEXO A. Datos suplementarios

\begin{tabular}{|c|c|c|c|c|c|c|c|c|c|c|c|c|c|c|c|c|c|c|c|c|c|c|}
\hline $\begin{array}{|ll|}\frac{\alpha}{2} & \\
\vec{J} & \frac{\alpha}{z} \\
\frac{5}{b} & \\
\end{array}$ & & & & $\sim$ & & $\nabla$ & $r$ & & & $\bar{\sim}$ & $\stackrel{\sim}{\stackrel{\rho}{2}}$ & - & 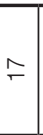 & $\stackrel{\infty}{-}$ & & $\sigma$ & 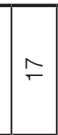 & $m$ & - & $-\stackrel{\infty}{\sim}$ & م & ন্ \\
\hline 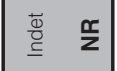 & & & & & & & & & & & & & & & & & & & & m & & m \\
\hline 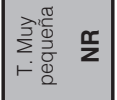 & & & & & & & & & & & & & & & & & & & & & & \\
\hline 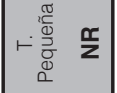 & & & & & & $m$ & - & & & \llcorner & $m$ & & 0 & م & & $\sim$ & & $-\sigma$ & & 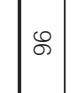 & م & 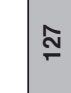 \\
\hline$\vdash \frac{\frac{\pi}{0}}{\frac{0}{2}} \frac{\frac{x}{z}}{z}$ & & & & & & - & - & & & 0 & ص & & $\nabla$ & \llcorner & & 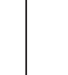 & - & - & - & $\overline{0}$ & & 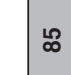 \\
\hline 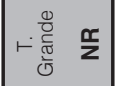 & & & & & & & . & & & $\diamond$ & م & & $\nabla$ & & & & & & & $\stackrel{\circ}{-1}$ & & న్ల \\
\hline 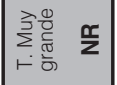 & & & & & & & & & & & & & & & & & & & & & & \\
\hline 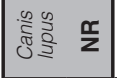 & & & & & & & & & & & & & & & & & & & & & & \\
\hline 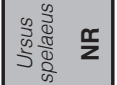 & & & & & & & & & & & & & & & & & & & & & & \\
\hline 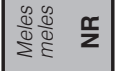 & & & & & & & & & & & & & & & & & & & & & & \\
\hline 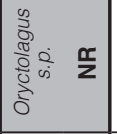 & & & & & & & & & & & & & & & & & & & & & & \\
\hline$\frac{\pi}{5} \frac{\pi}{0} \frac{\pi}{2}$ & & & & & & & & & & & & & & & & & & & & & & \\
\hline 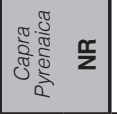 & & & & & & & & & & & & & & & & & & & & & & \\
\hline 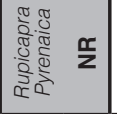 & & & & $r$ & & & & & & & & & & & & & & & & & & - \\
\hline 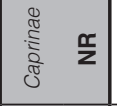 & & & & & & & & & & & & & & & & & & & & & & \\
\hline 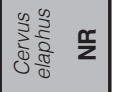 & & & & - & & & & & & 0 & $\sim$ & - & $\sim$ & $\wedge$ & & $\wedge$ & $\stackrel{2}{\circ}$ & - & & & & g \\
\hline$\frac{\mathfrak{w}}{3}$ & & & & & & & & & & & & & - & & & & & & & - & & - \\
\hline 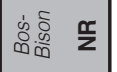 & & & & & & & & & & & & & & - & & & - & & & & & N \\
\hline & 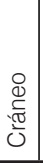 & $\approx$ & $\frac{\frac{\bar{\pi}}{\bar{\alpha}}}{\frac{\bar{x}}{4}}$ & $\begin{array}{l}\frac{0}{\bar{z}} \\
\frac{\bar{g}}{\bar{c}} \\
\frac{\bar{w}}{2}\end{array}$ & 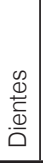 & , & 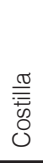 & 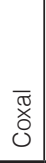 & \begin{tabular}{|l|}
$\frac{\pi}{3}$ \\
$\frac{2}{0}$ \\
0 \\
$w$ \\
$w$ \\
\end{tabular} & 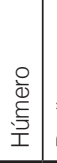 & 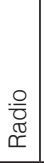 & $\frac{\sqrt[\pi]{5}}{5}$ & 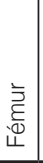 & . & 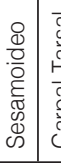 & 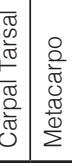 & 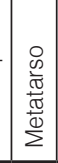 & \begin{tabular}{|c|c|} 
& \\
0 \\
0 \\
0 \\
$\frac{.0}{010}$ \\
$\frac{0}{2}$
\end{tabular} & 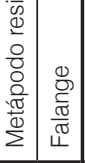 & 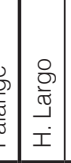 & 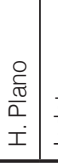 & 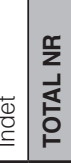 \\
\hline
\end{tabular}




\begin{tabular}{|c|c|c|c|c|c|c|c|c|c|}
\hline & \multirow{2}{*}{ NR } & \multirow{2}{*}{$\%$ NRt } & \multicolumn{4}{|c|}{ Disposición } & \multicolumn{3}{|c|}{ Cara del hueso } \\
\hline & & & Aislado & Concentrado & Disperso & Generalizado & Cortical & Medular & Cortical y medular \\
\hline Descamación y exfoliación & & & $5,21 \%$ & $2,81 \%$ & $17,51 \%$ & $74,37 \%$ & $18,07 \%$ & $1,56 \%$ & $87,37 \%$ \\
\hline Trampling & 996 & $77^{\prime} 45 \%$ & $9,13 \%$ & $0,80 \%$ & $76,13 \%$ & $11,43 \%$ & & & \\
\hline Fisuras & 941 & $73,17 \%$ & $3,61 \%$ & $2,77 \%$ & $81,51 \%$ & $11,05 \%$ & $10,10 \%$ & $11,05 \%$ & $78,85 \%$ \\
\hline Redondeo & 592 & $46,03 \%$ & $4,06 \%$ & $3,38 \%$ & $26,35 \%$ & $66,21 \%$ & $5,57 \%$ & $9,97 \%$ & $84,46 \%$ \\
\hline Pulido & 1031 & $80,17 \%$ & $1,17 \%$ & $0,19 \%$ & $41,20 \%$ & $57,44 \%$ & $9,99 \%$ & $1,07 \%$ & $88,94 \%$ \\
\hline Manganeso & 1145 & $89,03 \%$ & $3,23 \%$ & $1,32 \%$ & $86,28 \%$ & $9,17 \%$ & $4,98 \%$ & $3,32 \%$ & $91,62 \%$ \\
\hline Concreción & 77 & $5,99 \%$ & $25,97 \%$ & $6,49 \%$ & $59,74 \%$ & $7,79 \%$ & $11,69 \%$ & $48,05 \%$ & $40,26 \%$ \\
\hline Vermiculaciones & 422 & $32 \%$ & $26,33 \%$ & $3,43 \%$ & $68,19 \%$ & $1,83 \%$ & $68,72 \%$ & $7,08 \%$ & $24,20 \%$ \\
\hline Corrosión & 422 & $32 \%$ & $6,90 \%$ & $2,86 \%$ & $88,34 \%$ & $1,90 \%$ & $72,51 \%$ & $3,08 \%$ & $24,41 \%$ \\
\hline
\end{tabular}

Tabla A.2: Conjunto de procesos tafonómicos que afectan al registro estudiado. Se recoge tanto el número de restos en los que se encuentra (NR) y su proporción con respecto al total (\%NRt), como su disposición y la cara del hueso en la que se encuentra. Cabe reseñar que un 0,08\% de los manganesos se han encontrado en el paño de fractura. / Set of taphonomic processes that affect the registry studied. It includes both the number of remains in which it is (NR) and its proportion with respect to the total (\% NRt), its disposition and the face of the bone in which it is found. It should be noted that $0.08 \%$ of the manganese have been found in the fracture cloth.

\begin{tabular}{|l|l|l|l|}
\hline & & With Rows & With Columns \\
\hline Statistic & Symmetric & Dependent & Dependent \\
\hline Lambda & 0,0000 & 0,0000 & 0,0000 \\
\hline Uncertainty Coeff. & 0,0070 & 0,0056 & 0,0092 \\
\hline Somer's D & 0,0605 & 0,0811 & 0,0482 \\
\hline Eta & & 0,0834 & 0,0717 \\
\hline
\end{tabular}

\begin{tabular}{|l|l|l|l|}
\hline & & With Rows & With Columns \\
\hline Statistic & Symmetric & Dependent & Dependent \\
\hline Lambda & 0,0000 & 0,0000 & 0,0000 \\
\hline Uncertainty Coeff. & 0,0037 & 0,0038 & 0,0036 \\
\hline Somer's D & 0,0388 & 0,0362 & 0,0418 \\
\hline Eta & & 0,0593 & 0,0583 \\
\hline
\end{tabular}

\begin{tabular}{|l|l|l|l|}
\hline Statistic & Value & P-Value & $D f$ \\
\hline Contingency Coeff. & 0,1074 & & \\
\hline Cramer's V & 0,0764 & & \\
\hline Conditional Gamma & 0,1402 & & \\
\hline Pearson's R & 0,0670 & 0,0000 & 4804 \\
\hline Kendall's Tau b & 0,0625 & 0,0000 & \\
\hline Kendall's Tau c & 0,0403 & & \\
\hline
\end{tabular}

\begin{tabular}{|l|l|l|l|}
\hline Statistic & Value & P-Value & Df \\
\hline Contingency Coeff. & 0,0867 & & \\
\hline Cramer's V & 0,0615 & & \\
\hline Conditional Gamma & 0,0646 & & \\
\hline Pearson's R & 0,0430 & 0,0029 & 4804 \\
\hline Kendall's Tau b & 0,0389 & 0,0029 & \\
\hline Kendall's Tau c & 0,0350 & & \\
\hline
\end{tabular}

\begin{tabular}{|l|l|l|l|}
\hline & & With Rows & With Columns \\
\hline Statistic & Symmetric & Dependent & Dependent \\
\hline Lambda & 0,0000 & 0,0000 & 0,0000 \\
\hline Uncertainty Coeff. & 0,0038 & 0,0022 & 0,0148 \\
\hline Somer's D & 0,0194 & 0,0926 & 0,0108 \\
\hline Eta & & 0,0331 & 0,0630 \\
\hline
\end{tabular}

\begin{tabular}{|l|l|l|l|}
\hline Statistic & Value & P-Value & Df \\
\hline Contingency Coeff. & $\mathbf{0 , 0 6 2 9}$ & & \\
\hline Cramer's V & $\mathbf{0 , 0 6 3 0}$ & & \\
\hline Conditional Gamma & $\mathbf{0 , 1 7 6 0}$ & & \\
\hline Pearson's R & $\mathbf{0 , 0 3 3 1}$ & 0,0218 & 4804 \\
\hline Kendall's Tau b & $\mathbf{0 , 0 3 1 7}$ & 0,0219 & \\
\hline Kendall's Tau c & $\mathbf{0 , 0 1 2 1}$ & & \\
\hline
\end{tabular}

Fig. A1. Sumario estadístico de la tabla de contingencia para la variable ángulo (Arriba izquierda), delineación (arriba derecha) y superficie (abajo izquierda). Se considera estadísticamente significativo con una P menor de 0'05 con un intervalo de confianza del 95\%. / Statistical summary of the contingency table for the variable angle (top left), delineation (top right) and surface (bottom left). It is considered statistically significant with a P less than 0.05 with a $95 \%$ confidence interval. 
